\title{
SELF-CALIBRATION OF SENSOR NETWORKS
}

\author{
A Thesis \\ Presented in Partial Fulfillment of the Requirements for \\ the Degree Master of Science in the \\ Graduate School of The Ohio State University \\ By
}

Robert Matthew Patterson, B.S.

$* * * * *$

The Ohio State University

2002

Master's Examination Committee:

Approved by

Randolph L. Moses, Adviser

Lee C. Potter

Adviser

Department of Electrical

Engineering 


\begin{abstract}
Unattended sensor networks are becoming increasingly valuable for many military and commercial applications. A number of sensors are distributed in a region of interest. These sensors have the ability to sense and record energy, process data, and communicate with a central information processor. The information gained from signal processing is often used for detecting, tracking, and identifying objects of interest.

In certain circumstances, location and orientation information regarding these sensors is unknown after being placed in the scene. The problem considered in this thesis is how to locate and orient these sensors. We present methods for solving this problem using calibration source signals. Sources are distributed in the same region of interest, with their locations and signal emission times being unknown. Each sensor has the ability to generate direction-of-arrival (DOA) and time-of-arrival (TOA) estimates from the source signals. The goal is to estimate the locations and orientations of all sensors using these TOA and DOA measurements.
\end{abstract}

We develop necessary conditions for solving the self-calibration problem and provide a maximum likelihood solution and corresponding location error estimate. A lower bound on calibration accuracy via the Cramér-Rao Bound is found. We also consider the problem of locating and orienting a network of unattended sensors using nominal location information in the form of a prior probability distribution function. We develop a Bayes approach to the 
calibration problem and compute accuracy bounds on the calibration procedure. A maximum a posteriori estimation algorithm is shown to achieve the accuracy bound. Results using both synthetic data and field measurements are presented. 
This thesis is dedicated to my parents 


\section{ACKNOWLEDGMENTS}

First and foremost, I would like to thank my advisor, Randolph L. Moses, for his advice and guidance. From beginning to end, I am grateful for everything he has helped me accomplish as a student at The Ohio State University. I would also like to thank my examination committee for their time and effort. I am grateful to the university for funding my education while at The Ohio State University . I am also grateful to Dushyanth Krishnamurthy for his help in introducing me to this research problem. I would like to thank Hiranya Parakrama for all of her support and encouragement. This thesis is dedicated to my parents in thanks for their endless love and support. 


\title{
VITA
}

\author{
July $6,1977 \ldots \ldots \ldots \ldots \ldots \ldots \ldots \ldots \ldots$ Born - West Chester, PA.

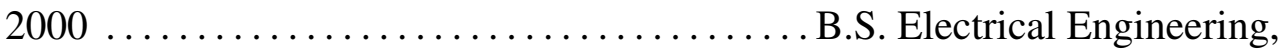 \\ Lafayette College, Easton, PA. \\ 2001-2002 ....................... Graduate Research Associate, \\ The Ohio State University, Columbus, \\ $\mathrm{OH}$.
}

\section{PUBLICATIONS}

\section{Research Publications}

R. Moses and R. Patterson, "Self-Calibration of Sensor Networks," Unattended Ground Sensor Technologies and Applications IV (Proceedings of SPIE Vol. 4743), April 2-5, 2002, Orlando, FL.

R. Moses, D. Krishnamurthy, and R. Patterson, "An Auto-Calibration Method for Unattended Ground Sensors," The 2002 International Conference on Acoustics, Speech, and Signal Processing (ICASSP-02)., May 13-17, 2002, Orlando, FL, Paper SAM-P02.04.

R. Moses, D. Krishnamurthy, and R. Patterson, "Self-Calibration of Unattended Ground Sensor Networks," Proceedings of the 2001 Battlefield Acoustic and Seismic Sensing Workshop, October 23-25, 2001, Columbia, MD.

R. Moses, R. Patterson, D. Krishnamurthy, N. Srour, and T. Pham, "Self-Calibration of Unattended Ground Sensor Networks," Proceedings of the Fifth Annual Federated Laboratory Symposium on Advanced Sensors, March 20-22, 2001, College Park, MD, pp. 63-68.

\section{FIELDS OF STUDY}


Major Field: Electrical Engineering 
TABLE OF CONTENTS

Page

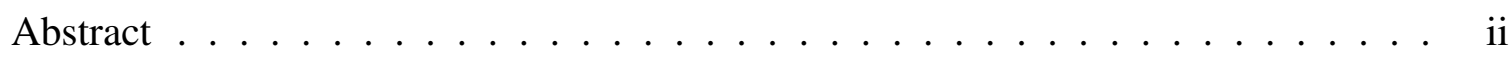

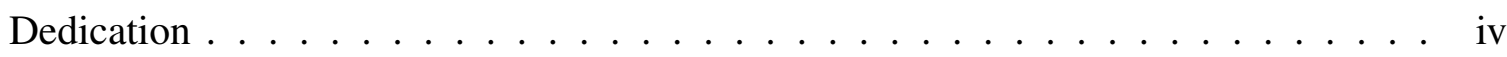

Acknowledgments ....................... . .

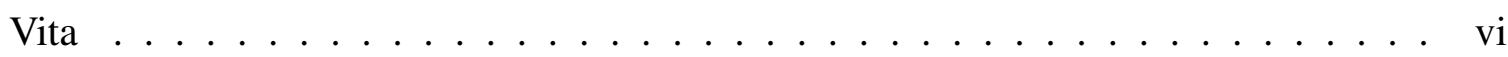

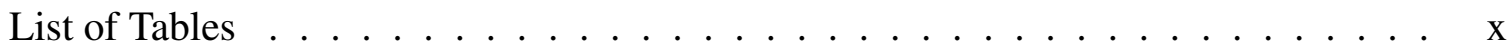

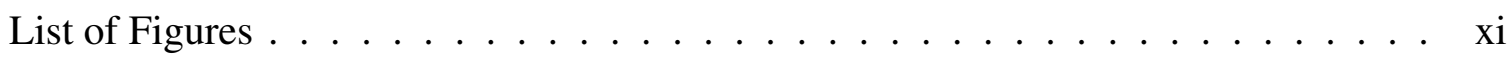

Chapters:

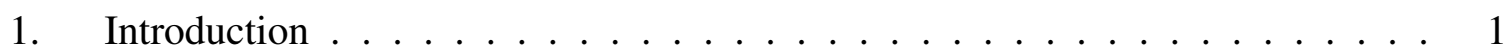

1.1 Networks of Sensors . . . . . . . . . . . . . . . . . . . 1

1.2 Sensor Self-Calibration . . . . . . . . . . . . . . . 3

1.3 Relation to Prior Work . . . . . . . . . . . . . . . 3

1.4 Contribution of this Thesis . . . . . . . . . . . . . . 5

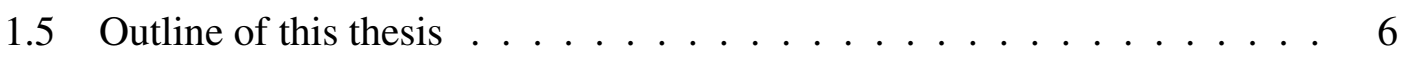

2. A Self-Localization Method for Wireless Sensor Networks . . . . . . . . . . 7

2.1 Introduction . . . . . . . . . . . . . . . . 7

2.2 Problem Statement and Notation . . . . . . . . . . . . . . . . . . 11

2.3 Existence and Uniqueness of Solutions . . . . . . . . . . . . . . . . . . . . . . . . . . 14

2.4 Maximum Likelihood Self-Calibration . . . . . . . . . . . . . . . 17

2.4.1 The Maximum Likelihood Estimate . . . . . . . . . . . . . . 18

2.4.2 Nonlinear Least Squares Solution . . . . . . . . . . . . . . . . . . . . 18

2.4.3 Estimation Accuracy . . . . . . . . . . . . . . . . . . 19

viii 
2.4 .4 Partial Measurements . . . . . . . . . . . . . . . . 20

2.5 Numerical Results . . . . . . . . . . . . . . . . . . . 22

2.5.1 Synthetic Data Example . . . . . . . . . . . . . . . 22

2.5.2 Field Test Results . . . . . . . . . . . . . . . . . . 27

2.6 Conclusions . . . . . . . . . . . . . . . . . . 30

3. Self-Calibration of Sensor Networks . . . . . . . . . . . . . 33

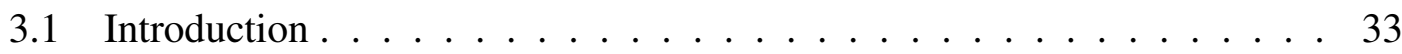

3.2 Self-Localization Problem Formulation . . . . . . . . . . . . . 36

3.2.1 Absolute and Relative Calibration . . . . . . . . . . . . . . 37

3.2.2 Calibration Measurements . . . . . . . . . . . . . . . . . . . . . . . . . . . 48

3.3 Bayesian Self-Calibration . . . . . . . . . . . . . . . . . 40

3.3.1 Gaussian Measurement Uncertainty . . . . . . . . . . . . . . . . 40

3.4 Algorithms for Self-Calibration . . . . . . . . . . . . . . . . . . . . . . . . . 41

3.4.1 Uncertainty in Propagation Velocity . . . . . . . . . . . . . . . 43

3.5 Estimation Accuracy . . . . . . . . . . . . . . . . . . . . . . 44

3.5.1 Estimation Accuracy of Relative Location . . . . . . . . . . . . 44

3.5.2 Accuracy of Maximum A Posteriori Absolute Location Estimate 46

3.6 Experimental Results . . . . . . . . . . . . . . . . . . . . 46

3.6.1 Synthetic Data Example . . . . . . . . . . . . . . . 46

3.6 .2 Field Test Results . . . . . . . . . . . . . . . . . 50

3.7 Conclusions . . . . . . . . . . . . . . . . . 51

4. Graphical User Interface . . . . . . . . . . . . . . . . . 54

4.1 Program Description . . . . . . . . . . . . . . . . . 54

4.2 Sensor and Source Placement and Initialization . . . . . . . . . . . 55

4.3 Cramér-Rao Bound and Maximum Likelihood Estimation . . . . . . . . 56

4.4 Additional Features . . . . . . . . . . . . . . . . . . . 57

5. Contributions and Future Work . . . . . . . . . . . . . . . . 58

5.1 Conclusions . . . . . . . . . . . . . . . . 58

5.2 Ideas for Future Work . . . . . . . . . . . . . . . . . . 59

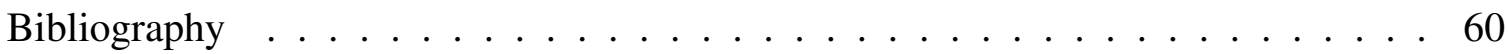




\section{LIST OF TABLES}

Table

2.1 Minimal Solutions for Sensor Self-Localization. . . . . . . . . . . . . . 16

3.1 Average $2 \sigma$ uncertainty radius for relative and absolute sensor calibration for the example presented. . . . . . . . . . . . . . . . . . . 49 


\section{LIST OF FIGURES}

Figure

Page

2.1 Sensor network architecture. A number of low-cost sensors are deployed in a region. Each sensor communicates to a local CIP, which relays information to a more distant command center. . . . . . . . . . . . . 8

2.2 Sensor self-localization scenario. . . . . . . . . . . . . . . . . 10

2.3 A circular arc is the locus of possible sensor locations whose angle between two known points is constant. . . . . . . . . . . . . . . . . . 17

2.4 Example scene showing ten sensors (stars) and eleven sources (squares). Also shown are the $2 \sigma$ location uncertainty ellipses of the sensors and sources; these are on average less than $1 \mathrm{~m}$ in radius and show as small dots. The locations of sensors $A 1$ and $A 2$ are assumed to be known. . . . . 23

2.5 Two standard deviation location uncertainty ellipses for sensors $A 3$ and $A 9$ from Figure 2.4 . . . . . . . . . . . . . . . . . . . . 23

2.6 The $2 \sigma$ location uncertainty ellipses for the scene in Figure 2.4 when the location and orientation of sensor $A 1$ is assumed to be known. . . . . . . . 25

2.7 Average $2 \sigma$ location uncertainty radius for the scenes in Figures 2.4 and 2.6 as a function of the number of source signals used. . . . . . . . . . 2 26

2.8 Detection probability of a source a distance $r$ from a sensor, for three values

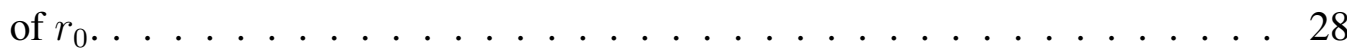

2.9 Average $2 \sigma$ location uncertainty for sensors in Figure 2.4 for three detection probability profiles. . . . . . . . . . . . . . . 28

2.10 Average number of sources detected by each sensor for sensors in Figure 2.4 for three detection probability profiles. . . . . . . . . . . . . 
2.11 Actual and estimated sensor locations, and estimated source locations, using field test data. Sensor A1 is assumed to have known location, and the angle from sensor A1 to sensor A4 is assumed known. Error distances between actual and estimated sensor locations are shown in parentheses. . . 31

3.1 Sensor network architecture. A network of low-cost sensors are deployed in a region. Each sensor communicates to a local Central Information Processor, which relays information to a higher-level information processing center. . . . . . . . . . . . . . . . . . 34

3.2 Sensor self-localization scenario. Sensors are placed in the field at unknown locations and orientations. Calibrations sources, also at unknown locations, generate signals time-of-arrival and direction-of-arrival measurements at the sensors are used for network self-calibration. . . . . . . . . . . 35

3.3 Top: Example scene showing ten sensors A1-A10 (stars) and eleven sources S1-S11 (squares). Also shown are the $2 \sigma$ location uncertainty ellipses of the sensors and sources; these are on average less than $0.5 \mathrm{~m}$ in radius and show as small dots. Bottom: enlarged views near sensors A6 and A8, showing $2 \sigma$ location uncertainty ellipses along with location estimates from 200 Monte-Carlo experiments. . . . . . . . . . . . . . . . . . . 48

3.4 Two standard deviation location uncertainty ellipses for sensors $A 6$ and $A 8$ from Figure $3.3 \ldots \ldots \ldots$

3.5 Actual and estimated sensor locations, and estimated source locations, using field test data. Error distances between actual and estimated sensor locations are shown in parentheses. . . . . . . . . . . . . 52

4.1 A screen shot of the auto-calibration demonstration's main display. . . . . . 55 


\section{CHAPTER 1}

\section{INTRODUCTION}

\subsection{Networks of Sensors}

Advancements in sensor network technology in recent years have led to a growth in the use of these devices. Networks of sensors are being applied to many commercial and military applications $[1,2,3,4]$. Typical application objectives include detecting, tracking, and/or identifying items of interest and physical or environmental monitoring $[1,4]$. We refer to a sensor as being a transducer or group of transducers than can detect a signal (acoustic, seismic, magnetic, pressure, etc.) and convert it to a voltage measurement. A sensor network is a cluster of sensors that are located in the same area of interest. The sensors are intended to be low-powered, contain simple signal processing capabilities, and be able to communicate with a data fusion center.

Sensors must be low-powered due to power constraints, and often they go into a "sleep" mode until an object of interest activates the sensor. Signal processing capabilities within the sensor allow it to take raw data and turn it into high-value data. Transmitting raw data through the communications network on each sensor would quickly deplete the battery, but transmitting compressed information infrequently would allow efficient use of the battery. Having a network of sensors enables information from each sensor to be combined at the 
Central Information Processor (CIP) for enhanced processing capabilities. In addition, by using many sensors in the network, a high degree of redundancy is built into the system. A defective sensor or a sensor with a dead battery will fail to cripple a networked system because of this.

Sensor networks are being developed for a vast array of applications in both commercial and military realms. Military applications concentrate on detecting, tracking, and identifying targets of interest for increased battlefield awareness. Commercial systems tend to focus on environmental monitoring, such as atmospheric, wind, and temperature conditions. Several systems in use today are listed below.

1. Researchers at the Lawrence Livermore National Laboratory are exploring methods of using unmanned aerial vehicles to distribute, operate, and maintain sensors placed in rugged terrain. They have built a prototype correlated sensor network for detecting and tracking ground-delivered nuclear material called the Wide Area Tracking System (WATS). Another application for which they have developed networked sensors is the Joint Biological Remote Early Warning System (JBREWS). This system can be used to provide field troops with early warning of a biological attack [5].

2. L-3 Communications, Inc. have developed unattended ground sensor systems called REMBASS and REMBASS II for use in the battlefield. REMBASS sensors are placed near areas where troop or vehicle movement is probable. The sensors respond to seismic and acoustic disturbances, infrared energy, and magnetic field changes. Information is processed and sent in short, digital messages by VHF radio burst transmission. REMBASS II sensors incorporate advanced signal processing techniques to detect and validate the presence of targets [6]. 
3. Research and development by the Defense Advanced Research Projects Agency has led to a system of unattended ground sensors (UGS). Both Inter-netted UGS (IUGS) and miniature IUGS (MIUGS) are used to estimate the location and identification of ground and airborne targets [4].

4. Crossbow Technology, Inc. has developed a wireless sensor network for use in fighting forest fires. Their sensors can be dropped from airborne platforms to provide information on which direction the fire is spreading, thermal gradients, wind, humidity, and other variables helpful in fighting fires [7].

\subsection{Sensor Self-Calibration}

Upon deployment into a region of interest, sensors often either have unknown locations and orientations or these parameters are known with some degree of uncertainty. Highly accurate knowledge of each sensor's location and orientation is imperative for the sensor network to be reliable in tasks such as source localizing and tracking. A method of sensor self-calibration would be highly desirable in this case. Thus, much interest exists in the self-calibration of sensor networks using a minimal amount of additional hardware and processing.

\subsection{Relation to Prior Work}

Self-calibration of sensor networks is an active area of current research. A number of papers have studied sensor calibration to improve direction-of-arrival (DOA) estimation accuracy. Friedlander and Weiss consider direction finding in the presence of gain, phase, and mutual coupling uncertainties [8]. Calibration with respect to errors in location, phase, and gain is examined in [9]. 
Further research considers sensor calibration when nominal locations for the sensors are known. Weiss and Friedlander develop an iterative maximum likelihood approach for simultaneously estimating directions-of-arrival and sensor locations. $\mathrm{Ng}$ and See use a maximum likelihood approach to estimate unknown gain, phase, and mutual coupling as well as sensor position errors [10]. Flanagan and Bell present algorithms for improved sensor calibration with large errors in the nominal locations [11].

Other authors explore the use of beacon nodes for sensor localization. Beacon nodes are nodes that know their location and can broadcast this information to other nodes in the sensor network. Bulusu et al. use signals emitted by beacon nodes to estimate the position of unknown nodes [12]. Savvides et al. develop this idea further as an iterative multilateration technique, where unknown nodes, upon receiving information from beacon nodes and estimating their positions, become beacon nodes themselves [13]. The process is repeated iteratively until all nodes have known locations.

Cevher and McClellan perform sensor calibration using a single source travelling at a constant velocity [14]. Given DOA estimates from the sensors, target tracks can be generated with an extended Kalman filter. Properties of this filter allow relative sensor position and orientation calibration. Absolute calibration is achieved by knowing an absolute range to the target and a reference orientation.

Other research looks at finding source positions without estimating sensor positions. Yao et al. take a blind beamforming approach toward source localization [15]. With an array of randomly distributed sensors, a maximum power beam is formed to estimate source locations. Kaplan et al. derive maximum likelihood methods for source localization using only DOA measurements [16]. However, these methods assume all sensor locations are known. 
Much of the earlier work consider a sensor calibration problem that differs in at least one aspect from ours. In our paper we present relative and absolute calibration solutions using multiple source signals. We find algorithms to estimate sensor locations and orientations and source locations and emission times. Our algorithms are flexible and can include extra information if sensor or source parameters are known. Relative sensor calibration does not require any information to be known and can calibrate sources and sensors to within a translational and rotational ambiguity. Absolute calibration can be attained by either knowing location or orientation information about one or two sensors or sources, or by having nominal sensor positions given by uncertain prior location information.

\subsection{Contribution of this Thesis}

We present a method for localizing sensors placed in a field of interest using sources that emit calibration signals. The sensors detect these signals and can obtain direction-ofarrival (DOA) and time-of-arrival estimates (TOA). The sensors transmit these estimates to a central information processor which can find location and orientation estimates for the sensors.

Maximum likelihood (ML) solutions are derived for estimating unknown sensor and source parameters and are seeded with an initialization algorithm. We develop accuracy bounds on the location accuracy that is achievable by our ML estimates using the CramérRao Bound. We also address the issue of partial measurement of source signals by the sensors.

We present solutions to both a relative and absolute sensor calibration problem. Sensor calibration is viewed as a two-step process. First, we derive the solution to a relative calibration problem, which involves finding sensor and source parameter estimates with 
respect to a position and orientation centroid. Then, absolute calibration is used to fix the sensor/source scene with respect to some global map. We solve the absolute calibration problem in the case when some parameters may be known, and in the case when we have prior knowledge about the nominal positions of the sensors.

A demonstration of these calibration techniques is provided by a graphical user interface program. A user can place sensors and sources in a bounded scene of interest. The user can set several parameters as being known, or the user can view the sensor placement as being defined by some prior distribution. Accuracy bounds and maximum likelihood solutions can be calculated and examined by the user.

\subsection{Outline of this thesis}

The remainder of the thesis is organized in the following way. Chapter 2 considers sensor localization through use of calibration sources. Absolute calibration is obtained through the use of several known location and/or orientation parameters. Chapter 3 extends the work of Chapter 2 by incorporating prior knowledge about sensor locations when solving the absolute calibration problem. Chapter 2 has been submitted for publication to the Eurasip Journal on Applied Signal Processing in November, 2001, and Chapter 3 has appeared in the April 2002 SPIE Aerospace Sensing Symposium (minor corrections have been made). Chapter 4 describes a graphical user interface program that demonstrates these localization methods. Chapter 5 summarizes the results of the thesis and gives suggestions for future work. 


\section{CHAPTER 2}

\section{A SELF-LOCALIZATION METHOD FOR WIRELESS SENSOR NETWORKS}

\subsection{Introduction}

Unattended sensor networks are becoming increasingly important in a large number of military and civilian applications $[1,2,4]$. The basic concept is to deploy a large number of low-cost, self-powered sensors that acquire and process data. The sensors may include one or more acoustic microphones as well as seismic, magnetic, or imaging sensors. A typical sensor network objective is to detect, track, and classify objects in the neighborhood of the sensors.

We consider a sensor deployment architecture as shown in Figure 2.1. A number of lowcost sensors, each equipped with a local processor, a low-power communication transceiver, and one or more sensing capabilities, is set out in a planar region. Each sensor monitors its environment to detect, track, and characterize signatures. The sensed data is processed locally, and the result is transmitted to a local Central Information Processor (CIP) through a low-power communication network. The CIP fuses sensor information and transmits the processed information to a higher-level processing center. 


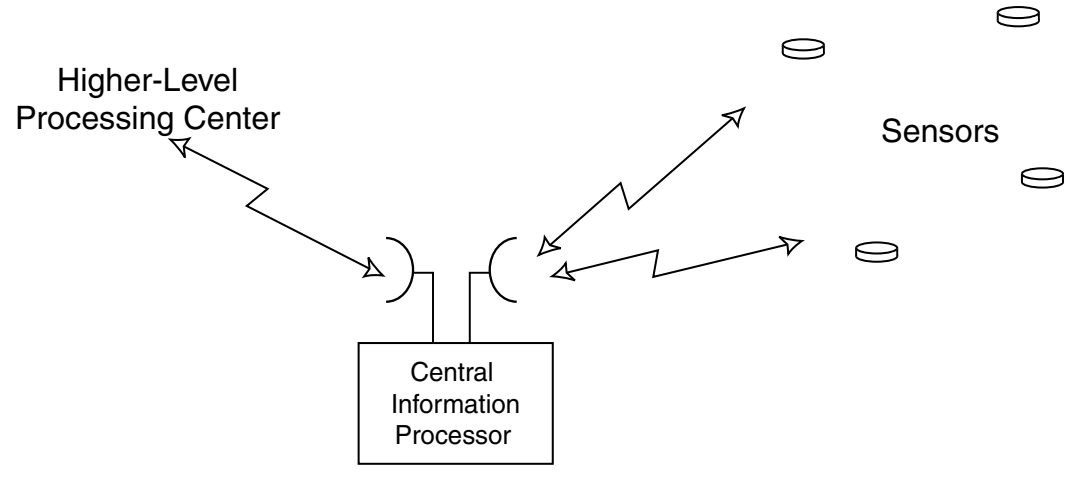

Figure 2.1: Sensor network architecture. A number of low-cost sensors are deployed in a region. Each sensor communicates to a local CIP, which relays information to a more distant command center.

In order to fuse information acquired by individual sensors, we must know the location and orientation of each sensor. However, accurate knowledge of sensor locations and orientations is often not available. These sensors are placed in the field by persons, by an air drop, or by artillery launch. For careful hand placement, accurate location and orientation of the sensors can be assumed; however, for most other sensor deployment methods, it is difficult or impossible to know accurately the location and orientation of each sensor. One could equip every sensor with a GPS and compass to obtain location and orientation information, but this adds to the expense and power requirements of the sensor and may increase susceptibility to jamming. Thus, there is interest in developing methods to self-localize the sensor network with a minimum of additional hardware, processing, or communication.

Self-localization in sensor networks is an active area of current research (see, e.g., [17, 12] and the references therein). Iterative multilateration-based techniques are considered in [18]. Bulusu et al. consider low-cost localization methods that use a number of beacon signals at known locations [12, 19]. Research on blind beamforming considers a related problem of forming a maximum power beam to a source without computing the source 
locations [15]. Cevher and McClellan consider sensor network self-calibration using a single acoustic source that travels along a straight line [14]. Bearings-only localization methods for sensor networks are considered in [16].

The self-localization problem is related to the problem of calibration of both narrowband and broadband sensor arrays to improve direction-of-arrival estimation accuracy [9, $11,8,10,20,21]$. These papers typically assume knowledge of the nominal sensor locations and high (or perfect) signal coherence between the sensors, and these assumptions may not be satisfied for many sensor network applications. Kozick and Sadler [22] investigate the effect of coherence loss on sensor fusion performance and present experimental coherence results for well-separated microphone arrays.

In this paper we consider an approach to sensor network self-calibration using sources at unknown locations in the field. Thus, we relax the assumption that beacon signals at known locations are available. The approach entails placing a number of signal sources in the same region as the sensors (see Figure 2.2). Each source generates a signal that is detected by some of the sensors, and each sensor measures the time-of-arrival (TOA) and direction-of-arrival (DOA) of the source signal. From these measurements we estimate the unknown locations and orientations of the sensors. In general, neither the source locations nor their signal emission times are assumed to be known. If partial information is available, it can be incorporated into the estimation procedure to improve the accuracy of the calibration. We show that if at least three sensors and two sources are used, the relative locations and orientations of all sensors, as well as the locations and signal emission times of all sources, can be estimated. The calibration is computed except for an unknown translation and rotation of the entire source-signal scene, which cannot be estimated unless 
additional information is available. However, with modest additional location or orientation information of one or two sources, absolute location and orientation estimates can be obtained.

We consider optimal signal processing of the measured self-localization data. We derive the Cramér-Rao bound (CRB) on localization accuracy to provide a lower bound on any unbiased localization estimator. It is useful in determining the best-case localization accuracy for a given problem and can provide a baseline standard against which suboptimal localization methods can be measured. We also develop a maximum likelihood estimation procedure and show that it achieves the CRB for reasonable TOA and DOA measurement errors.

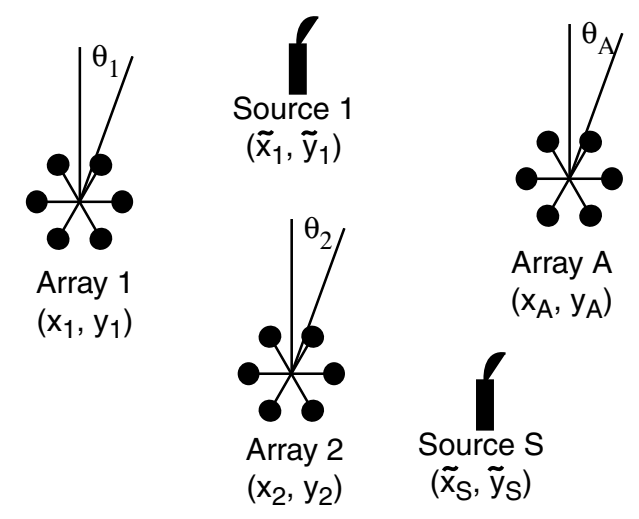

Figure 2.2: Sensor self-localization scenario.

There is a great deal of flexibility in the type of signal sources to be used. We require only that the times of arrival of the signals can be estimated by the sensors, say by matched filtering or (generalized) cross-correlation techniques [23, 24]. If the sensors can estimate signal arrival directions (as is the case with vector pressure sensors or arrays of aeroacoustic microphones), these estimates can be used to improve the calibration solution. Examples 
of source signals are short transients, pseudonoise-coded or direct-sequence waveforms, or pulsed signals. Since we do not assume knowledge of the signal location or emission time, signals of opportunity in the scene can also be used.

An outline of the chapter is as follows. In Section 2.2 we present a statement of the problem and justify our assumptions. In Section 2.3 we consider necessary conditions for a self-calibration solution and present methods for solving the self-calibration problem with a minimum number of sensors and sources. These methods provide initial estimates for an iterative descent computation needed to obtain maximum likelihood calibration parameter estimates derived in Section 2.4. Bounds on the calibration uncertainty are also derived. Section 2.5 presents numerical examples to illustrate the approach, and Section 2.6 presents conclusions and directions for future work.

\subsection{Problem Statement and Notation}

Assume we have a set of $A$ sensors in a plane, each with unknown location $\left\{a_{i}=\right.$ $\left.\left(x_{i}, y_{i}\right)\right\}_{i=1}^{A}$ and unknown orientation angle $\theta_{i}$ with respect to a reference direction (e.g., North). We consider the two-dimensional problem in which the sensors lie in a plane and the unknown reference direction is azimuth; an extension to the three-dimensional case is possible using similar techniques.

In the sensor field are also placed $S$ point source signals at locations $\left\{s_{j}=\left(\tilde{x}_{j}, \tilde{y}_{j}\right)\right\}_{j=1}^{S}$. The source locations are in general unknown. Each source emits a finite-length signal that begins at time $t_{j}$; the emission times are also in general unknown.

We initially assume each emitted source signal is detected by all of the sensors in the field and that each sensor measures the time-of-arrival and direction-of-arrival for that 
source (partial measurements are considered in Section 2.4.4). We denote the measured TOA and DOA at sensor $i$ of source $j$ as $t_{i j}$ and $\theta_{i j}$, respectively.

The DOA measurements are made with respect to a local (to the sensor) frame of reference. The absolute directions of arrival are not available because the orientation angle of each sensor is unknown (and must be estimated in the calibration procedure). Each source signal's time of arrival is measured with respect to a known, common time base. The time base can be established either by using the electronic communication network that links the sensors or by synchronizing the sensor processor clocks before deployment. The time base needs to be accurate to a number on the order of the sensor's time-of-arrival measurement uncertainty (1 msec in the examples considered in Section 5).

The set of $2 A S$ measurements are gathered in a vector

$$
X=\left[\begin{array}{c}
\operatorname{vec}(T) \\
\operatorname{vec}(\Theta)
\end{array}\right]^{T} \quad(2 A S \times 1)
$$

where $\operatorname{vec}(M)$ stacks the elements of a matrix $M$ columnwise and where

$$
T=\left[\begin{array}{cccc}
t_{11} & t_{12} & \ldots & t_{1 S} \\
t_{21} & t_{22} & \ldots & t_{2 S} \\
\vdots & \vdots & \ddots & \vdots \\
t_{A 1} & t_{A 2} & \ldots & t_{A S}
\end{array}\right], \quad \Theta=\left[\begin{array}{cccc}
\theta_{11} & \theta_{12} & \ldots & \theta_{1 S} \\
\theta_{21} & \theta_{22} & \ldots & \theta_{2 S} \\
\vdots & \vdots & \ddots & \vdots \\
\theta_{A 1} & \theta_{A 2} & \ldots & \theta_{A S}
\end{array}\right]
$$

Each sensor transmits its $2 S$ TOA and DOA measurements to a CIP, and these $2 A S$ measurements form the data with which the CIP computes the sensor calibration. Note that the communication cost to the CIP is low, and the calibration processing is entirely performed by the CIP. The CIP must be able to distinguish which measurements correspond to each source and which sensor transmitted each set of measurements. To indicate which measurements belong to a particular source, the sensor can be programmed to order the measurements in a predefined sequence. The CIP can determine which sensor transmitted each set of measurements in one of several ways. Each sensor can transmit at a different 
carrier frequency, a sensor identifier can be appended to the sequence of measurements, or the order of sensor transmission to the CIP can be preprogrammed with respect to the time-synchronized clock on each sensor.

Define the parameter vectors

$$
\begin{aligned}
\beta & =\left[x_{1}, y_{1}, \theta_{1}, \ldots, x_{A}, y_{A}, \theta_{A}\right]^{T} \quad(3 A \times 1) \\
\gamma & =\left[\tilde{x}_{1}, \tilde{y}_{1}, t_{1}, \ldots, \tilde{x}_{S}, \tilde{y}_{S}, t_{S}\right]^{T} \quad(3 S \times 1) \\
\alpha & =\left[\beta^{T}, \gamma^{T}\right]^{T} \quad(3(A+S) \times 1)
\end{aligned}
$$

Note that $\beta$ contains the sensor unknowns and $\gamma$ contains the source signal unknowns. We denote the true TOA and DOA of source signal $j$ at sensor $i$ as $\tau_{i j}(\alpha)$ and $\phi_{i j}(\alpha)$, respectively, and include their dependence on the parameter vector $\alpha$; they are given by:

$$
\begin{aligned}
\tau_{i j}(\alpha) & =t_{j}+\left\|a_{i}-s_{j}\right\| / c \\
\phi_{i j}(\alpha) & =\theta_{i}+\angle\left(a_{i}, s_{j}\right)
\end{aligned}
$$

where $a_{i}=\left[x_{i}, y_{i}\right]^{T}, a_{j}=\left[\tilde{x}_{j}, \tilde{y}_{j}\right]^{T},\|\cdot\|$ is the Euclidean norm, $\angle(\xi, \eta)$ is the angle between the points $\xi, \eta \in \mathcal{R}^{2}$, and $c$ is the signal propagation velocity.

Each element of $X$ has measurement uncertainty; we model the uncertainty as

$$
X=\mu(\alpha)+E
$$

where $\mu(\alpha)$ is the noiseless measurement vector whose elements are given by equations (2.6) and (2.7) for values of $i, j$ that correspond to the vector stacking operation in (2.1), and where $E$ is a random vector with known probability density function.

The self-calibration problem, then, is: given the measurement $X$, estimate $\beta$. The parameters in $\gamma$ are in general unknown and are nuisance parameters that must also be estimated. If some parameters in $\gamma$ are known, the complexity of the self-calibration problem is reduced, and the resulting accuracy of the $\beta$ estimate is improved. 


\subsection{Existence and Uniqueness of Solutions}

In this section we address the existence and uniqueness of solutions to the self-calibration problem and establish the minimum number of sensors and sources needed to obtain a solution. We assume that every sensor detects every source signal and computes both the TOA and DOA. In addition, we assume the TOA and DOA measurements are noiseless and correspond to values in a planar sensor-source scenario; that is, we assume they are solutions to (2.6) and (2.7) for some vector $\alpha \in \mathcal{R}^{3(A+S)}$. We establish the minimum number of sources and sensors needed to compute a unique calibration solution and give algorithms for finding the self-calibration solution in the minimal cases. These algorithms provide initial estimates to an iterative descent algorithm for the practical case of non-minimal, noisy measurements presented in Section 2.4.

The four cases below make different assumptions concerning what is known about the source signal locations and emission times. Of primary interest is the case where no source parameters are known; however, the solution for this case is based on solutions for cases in which partial information is available, so it is instructive to consider all four cases. In all four cases the number of measurements is $2 A S$, and determination of $\beta$ involves solving a nonlinear set of equations for its $3 A$ unknowns. Depending on the case considered, we may also need to estimate the unknown nuisance parameters in $\gamma$. The result for each case is summarized in Table 2.1.

Case 1: Known source locations and emission times. A unique solution for $\beta$ can be found for any number of sensors as long as there are $S \geq 2$ sources. In fact, the location and orientation of each sensor can be computed independently of other sensor measurements. The location of the $i$ th sensor, $a_{i}$, is found from the intersection of two circles with centers 
at the source locations and with radii $\left(t_{i 1}-t_{1}\right) / c$ and $\left(t_{i 2}-t_{2}\right) / c$. The intersection is in general two points; the correct location can be found using the sign of $\theta_{i 2}-\theta_{i 1}$. We note that the two circle intersections can be computed in closed form. Finally, from the known source and sensor locations and the DOA measurements, the sensor orientation $\theta_{i}$ can be uniquely found.

Case 2: Known source locations, unknown emission times. For $S \geq 3$ sources the location and orientation of each sensor can be computed in closed form independently of other sensors. A solution procedure is as follows. Consider the pair of sources $\left(s_{1}, s_{2}\right)$. Sensor $i$ knows the angle $\theta_{i 2}-\theta_{i 1}$ between these two sources. The set of all possible locations for sensor $i$ is an arc of a circle whose center and radius can be computed from the source locations (see Figure 2.3). Similarly, a second circular arc is obtained from the source pair $\left(s_{1}, s_{3}\right)$. The intersection of these two arcs is a unique point and can be computed in closed form. Once the sensor location is known, its orientation $\theta_{i}$ is readily computed from one of the three DOA measurements.

A solution for Case 2 can also be found using $S=2$ sources and $A=2$ sensors. The solution requires a one-dimensional search of a parameter over a finite interval. The known location of $s_{1}$ and $s_{2}$ and the known angle $\theta_{11}-\theta_{12}$ means that sensor $a_{1}$ must lie on a known circular arc, as in Figure 2.3. Each location along the arc determines the source emission times $t_{1}$ and $t_{2}$. These emission times are consistent with the measurements from the second sensor for exactly one position $a_{1}$ along the arc.

Case 3: Unknown source locations, known emission times. In this case and in Case 4 below, the calibration problem can only be solved to within an unknown translation and rotation of the entire sensor-source scene because any translation or rotation of the entire 


\begin{tabular}{|c|c|c|c|}
\hline Case & \# Unknowns & Minimum $A, S$ & Comments \\
\hline $\begin{array}{c}\text { Known Locations } \\
\text { Known Times }\end{array}$ & $3 A$ & $A=1, S=2$ & closed form solution \\
\hline $\begin{array}{c}\text { Known Locations } \\
\text { Unknown Times }\end{array}$ & $3 A+S$ & $A=1, S=3$ & closed form solution \\
\cline { 2 - 4 } & $3 A+S$ & $A=2, S=2$ & 1-D iterative solution \\
\hline $\begin{array}{c}\text { Unknown Locations } \\
\text { Known Times }\end{array}$ & $3(A-1)+2 S$ & $\begin{array}{c}A=2, S=2 \\
\text { closed form solution }\end{array}$ \\
\hline $\begin{array}{c}\text { Unknown Locations } \\
\text { Unknown Times }\end{array}$ & $3(A+S-1)$ & $\begin{array}{c}A=2, S=3 \\
\text { or } \\
A=3, S=2\end{array}$ & 2-D iterative solution \\
\hline
\end{tabular}

Table 2.1: Minimal Solutions for Sensor Self-Localization.

scene does not change the $t_{i j}$ and $\theta_{i j}$ measurements. To eliminate this ambiguity, we assume the location and orientation of the first sensor are known; without loss of generality we set $x_{1}=y_{1}=\theta_{1}=0$. We solve for the remaining $3(A-1)$ parameters in $\beta$.

For the case of unknown source locations, a unique solution for $\beta$ is computable in closed form for $S=2$ and any $A \geq 2$ (the case $A=1$ is trivial). From sensor $a_{1}$ the range to each source can be computed as $r_{j}=\left(t_{1 j}-t_{j}\right) / c$. Since its bearing is known as well, the locations of the two sources can be found. The locations and orientations of the remaining sensors are then computed using the method of Case 1.

Case 4: Unknown source locations and emission times. For this case it can be shown that an infinite number of calibration solutions exists for $A=S=2,{ }^{1}$ but a unique solution exists in almost all cases for either $A=2, S=3$ or $A=3, S=2$. Although we do not know of a case for which the $\beta$ parameters cannot be uniquely found, in some degenerate cases not all of the $\gamma$ parameters can be uniquely determined.

${ }^{1}$ Note that for $A=S=2$ there are 8 measurements and 9 unknown parameters. The set of possible solutions in general lies on a one-dimensional manifold in the 9-dimensional parameter space. 


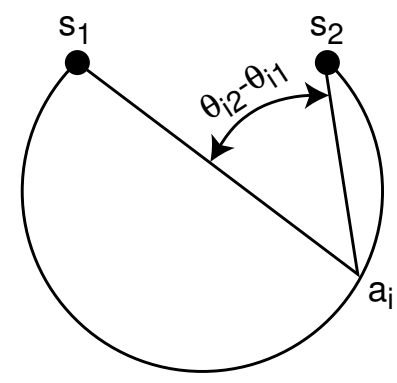

Figure 2.3: A circular arc is the locus of possible sensor locations whose angle between two known points is constant.

Closed form calibration solutions are not known for this case, but solutions that require a two-dimensional search can be found. We outline one such solution that works for either $A=2$ and $S \geq 3$ or $S=2$ and $A \geq 3$. Assume as before that sensor $a_{1}$ is at location $\left(x_{1}, y_{1}\right)=(0,0)$ with orientation $\theta_{1}=0$. If we know the two source emission times $t_{1}$ and $t_{2}$, we can find the locations of sources $s_{1}$ and $s_{2}$ as in Case 3. From the two known source locations, all remaining sensor locations and orientations can be found using the procedure in Case 1, and then all remaining source locations can be found using triangulation from the known arrival angles and known sensor locations. These solutions will be inconsistent except for the correct values of $t_{1}$ and $t_{2}$. The calibration procedure, then, is to iteratively adjust $t_{1}$ and $t_{2}$ to minimize the error between computed and measured time delays and arrival angles.

\subsection{Maximum Likelihood Self-Calibration}

In this section we derive a maximum likelihood (ML) estimator for the unknown sensor location and orientation parameters. The algorithm involves the solution of a set of nonlinear equations for the unknown parameters, including the unknown nuisance parameters in 
$\gamma$. The solution is found by iterative minimization of a cost function; we use the methods in Section 3 to initialize the iterative descent. In addition, we derive the Cramér-Rao Bound (CRB) for the variance of the unknown parameters in $\alpha$; the CRB also gives high-SNR parameter variance of the ML parameter estimates.

\subsubsection{The Maximum Likelihood Estimate}

We assume the measurement uncertainty $E$ in equation (2.8) is Gaussian with zero mean and known covariance $\Sigma$. In this case the likelihood function is

$$
\begin{aligned}
f(X ; \alpha) & =\frac{1}{(2 \pi)^{A S}|\Sigma|^{1 / 2}} \exp \left\{-\frac{1}{2} Q(X ; \alpha)\right\} \\
Q(X ; \alpha) & =[X-\mu(\alpha)]^{T} \Sigma^{-1}[X-\mu(\alpha)]
\end{aligned}
$$

A special case is when the measurement errors are uncorrelated and the TOA and DOA measurement errors have variances $\sigma_{t}^{2}$ and $\sigma_{\theta}^{2}$, respectively; equation (2.10) then becomes

$$
Q(X ; \alpha)=\sum_{i=1}^{A} \sum_{j=1}^{S}\left[\frac{\left(t_{i j}-\tau_{i j}(\alpha)\right)^{2}}{\sigma_{t}^{2}}+\frac{\left(\theta_{i j}-\phi_{i j}(\alpha)\right)^{2}}{\sigma_{\theta}^{2}}\right]
$$

Depending on the particular knowledge about the source signal parameters, none, some, or all of the parameters in $\alpha$ may be known. We let $\alpha_{1}$ denote the vector of unknown elements of $\alpha$, and let $\alpha_{2}$ denote the vector of known elements in $\alpha$. Using this notation along with equation (2.9), the maximum likelihood estimate of $\alpha_{1}$ is

$$
\hat{\alpha}_{1, M L}=\arg \max _{\alpha_{1}} f\left(X, \alpha_{2} ; \alpha\right)=\arg \min _{\alpha_{1}} Q(X ; \alpha)
$$

\subsubsection{Nonlinear Least Squares Solution}

The solution of (2.12) involves solving a nonlinear least squares problem. A standard iterative descent procedure can be used, initialized using one of the solutions in Section 3. In our implementation we used the Matlab function lsqnonlin. 
The straightforward nonlinear least squares solution we adopted converged quickly (in several seconds for all examples tested) and displayed no symptoms of numerical instability; however, alternative methods for solving equation (2.12) may reduce computation. For example, one can divide the parameter set and iterate first on the sensor location parameters and second on the remaining parameters. Although the sensor orientations and source parameters depend nonlinearly on the sensor locations, computationally efficient approximations exist (see, e.g., [25]), so the computational savings of lower dimensional searches may exceed the added computational cost of iterations nested in iterations if the methods are tuned appropriately. Similarly, one can view the source parameters as nuisance parameters and employ estimate-maximize (EM) algorithms to obtain the ML solution [26].

\subsubsection{Estimation Accuracy}

The Cramér-Rao Bound (CRB) gives a lower bound on the covariance of any unbiased estimate of $\alpha_{1}$. It is a tight bound in the sense that $\hat{\alpha}_{1, M L}$ has parameter uncertainty given by the CRB for high signal-to-noise ratio; that is, $\operatorname{as}_{\max } \Sigma_{i i} \rightarrow 0$. Thus, the CRB is a useful tool for analyzing calibration uncertainty.

The CRB can be computed from the Fisher Information Matrix of $\alpha_{1}$. The Fisher Information Matrix is given by [24]

$$
I_{\alpha_{1}}=E\left\{\left[\nabla_{\alpha_{1}} \ln f(T, \Theta ; \alpha)\right]\left[\nabla_{\alpha_{1}} \ln f(T, \Theta ; \alpha)\right]^{T}\right\}
$$

The partial derivatives are readily computed from equations (2.9), (2.6), and (2.7); we find that

$$
I_{\alpha_{1}}=\left[G^{\prime}\left(\alpha_{1}\right)\right]^{T} \Sigma^{-1}\left[G^{\prime}\left(\alpha_{1}\right)\right]
$$

where $G^{\prime}\left(\alpha_{1}\right)$ is the $2 A S \times \operatorname{dim}\left(\alpha_{1}\right)$ matrix whose $i j$ th element is $\partial \mu_{i}\left(\alpha_{1}\right) / \partial\left(\alpha_{1}\right)_{j}$. 
For Cases 3 and 4, the Fisher Information Matrix is rank deficient due to the translational and rotational ambiguity in the self-calibration solution in those two cases. One solution is to assume some of the sensor or source parameters are known. Examples are to assume known location and orientation of a single sensor, or to assume known location of two sensors or sources. These assumptions might be realized by equipping one sensor with a GPS and a compass, or by equipping two sensors or sources with GPSs. Let $\tilde{\alpha}_{1}$ denote the vector obtained by removing these assumed known parameters from $\alpha_{1}$. To compute the CRB matrix for $\tilde{\alpha}_{1}$ in this case, we first remove all rows and columns in $I_{\alpha_{1}}$ that correspond to the assumed known parameters and then invert the remaining matrix [24]:

$$
C_{\tilde{\alpha}_{1}}=\left[I_{\tilde{\alpha}_{1}}\right]^{-1}
$$

\subsubsection{Partial Measurements}

So far we have assumed that every sensor detects and measures both the TOA and DOA from every source signal. In this section we relax that assumption. We assume that each emitted source signal is detected by only a subset of the sensors in the field and that a sensor that detects a source may measure the TOA and/or the DOA for that source, depending on its capabilities. We denote the availability of a measurement using two indicator functions $I_{i j}^{t}$ and $I_{i j}^{\theta}$, where

$$
I_{i j}^{t}, I_{i j}^{\theta} \in\{0,1\}
$$

If sensor $i$ measures the TOA (DOA) for source $j$, then $I_{i j}^{t}=1\left(I_{i j}^{\theta}=1\right)$; otherwise, the indicator function is set to zero. Furthermore, let $L$ denote the $2 A S \times 1$ vector whose $k$ th element is 1 if $X_{k}$ is measured and is 0 if $X_{k}$ is not measured; $L$ is thus obtained by forming $A \times S$ matrices $I^{t}$ and $I^{\theta}$ and stacking their columns into a vector as in equation (2.1). 
Finally, define $\tilde{X}$ to be the vector formed from elements of $X$ for which measurements are available, so $X_{k}$ is in $\tilde{X}$ if $L_{k}=1$.

The maximum likelihood estimator for the partial measurement case is similar to equation (2.12) but uses only those elements of $X$ for which the corresponding element of $L$ is one. Thus,

$$
\hat{\alpha}_{1, M L}=\arg \min _{\alpha_{1}} \tilde{Q}(\tilde{X} ; \alpha)
$$

where (assuming uncorrelated measurement errors as in equation (2.11)),

$$
\tilde{Q}(\tilde{X} ; \alpha)=\sum_{i=1}^{A} \sum_{j=1}^{S}\left[\frac{\left(t_{i j}-\tau_{i j}(\alpha)\right)^{2}}{\sigma_{t}^{2}} I_{i j}^{t}+\frac{\left(\theta_{i j}-\phi_{i j}(\alpha)\right)^{2}}{\sigma_{\theta}^{2}} I_{i j}^{\theta}\right]
$$

The Fisher Information Matrix for this case is similar to equation (2.13), but includes only information from available measurements; thus

$$
\tilde{I}_{\alpha_{1}}=\left[\tilde{G}^{\prime}\left(\alpha_{1}\right)\right]^{T} \Sigma^{-1}\left[\tilde{G}^{\prime}\left(\alpha_{1}\right)\right]
$$

where

$$
\left[\tilde{G}^{\prime}\left(\alpha_{1}\right)\right]_{i j}=L_{i} \cdot \frac{\partial \mu_{i}\left(\alpha_{1}\right)}{\partial\left(\alpha_{1}\right)_{j}}
$$

The above expression readily extends to the case when the probability of sensor $i$ detecting source $j$ is neither zero or one. If $\Sigma$ is diagonal, the FIM for this case is given by

$$
I_{\alpha_{1}}=\left[G^{\prime}\left(\alpha_{1}\right)\right]^{T} \Sigma^{-1} P_{D}\left[G^{\prime}\left(\alpha_{1}\right)\right]
$$

where $P_{D}$ is a diagonal matrix whose $k$ th diagonal element is the probability that measurement $X_{k}$ is available.

We note that when partial measurements are available, the ML calibration may not be unique. For example, if only TOA measurements are available, a scene calibration 
solution and its mirror image have the same likelihoods. A complete understanding of the uniqueness properties of solutions in the partial measurement case is a topic of current research.

\subsection{Numerical Results}

This section presents numerical examples of the self-calibration procedure. First, we present a synthetically-generated example consisting of ten sensors and 2-11 sources placed randomly in a $2 \mathrm{~km} \times 2 \mathrm{~km}$ region. Second, we present results from field measurements using acoustic sources and sensors.

\subsubsection{Synthetic Data Example}

We consider a case in which ten sensors are randomly placed in a $2 \mathrm{~km} \times 2 \mathrm{~km}$ region. In addition, between two and 11 sources are randomly placed in the same region. The sensor orientations and source emission times are randomly chosen. Figure 2.4 shows the locations of the sensors and sources. We initially assume every sensor detects each source emission and measures the TOA and DOA of the source. The measurement uncertainties are Gaussian with standard deviations of $\sigma_{t}=1 \mathrm{msec}$ for the TOAs and $\sigma_{\theta}=3^{\circ}$ for the DOAs. Neither the locations nor emission times of the sources are assumed to be known. In order to eliminate the translation and rotation uncertainty in the scene, we assume either two sensors have known locations or one sensor has known location and orientation.

Figure 2.4 also shows the two standard deviation $(2 \sigma)$ location uncertainty ellipses for both the sources and sensors assuming the locations of sensors $A 1$ and $A 2$ are known. The ellipses are obtained from the $2 \times 2$ covariance submatrices of the CRB in equation (2.14) that correspond to the location parameters of each sensor or source. These ellipses appear as small dots in the figure; enlarged views for two sensors are shown in Figure 2.5. 


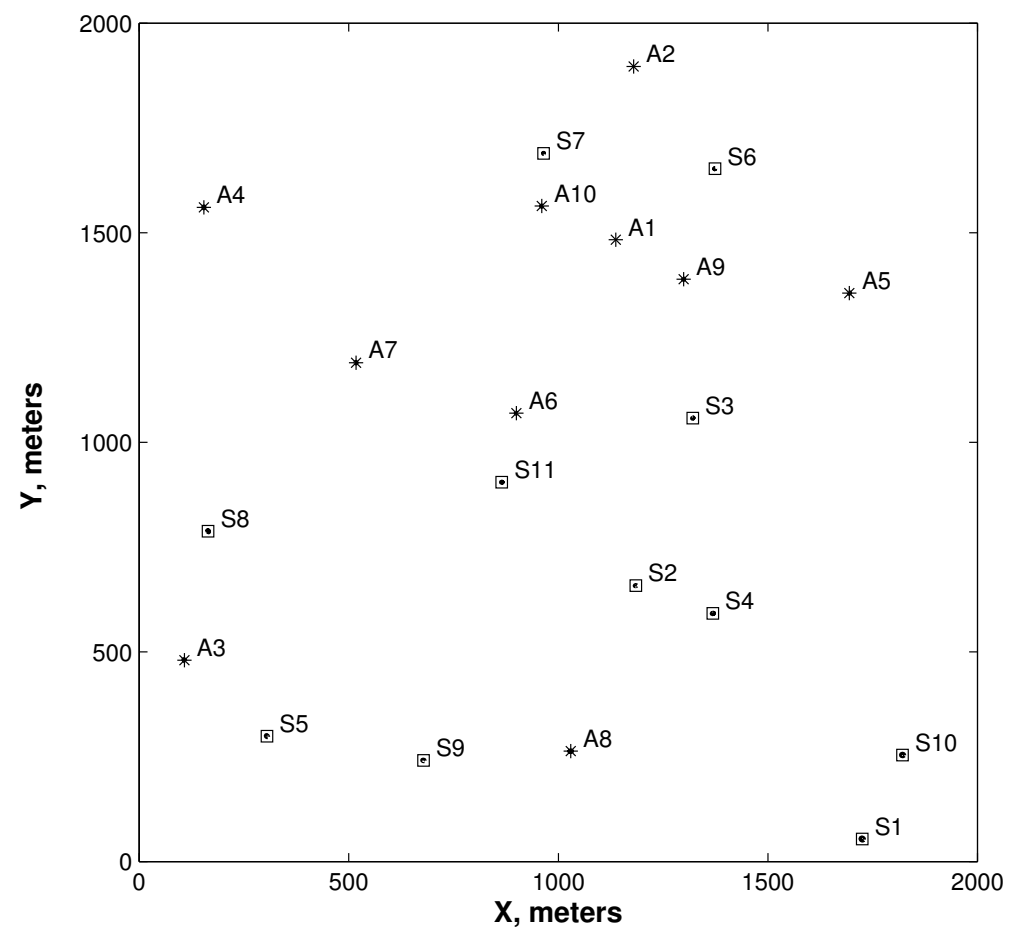

Figure 2.4: Example scene showing ten sensors (stars) and eleven sources (squares). Also shown are the $2 \sigma$ location uncertainty ellipses of the sensors and sources; these are on average less than $1 \mathrm{~m}$ in radius and show as small dots. The locations of sensors $A 1$ and $A 2$ are assumed to be known.
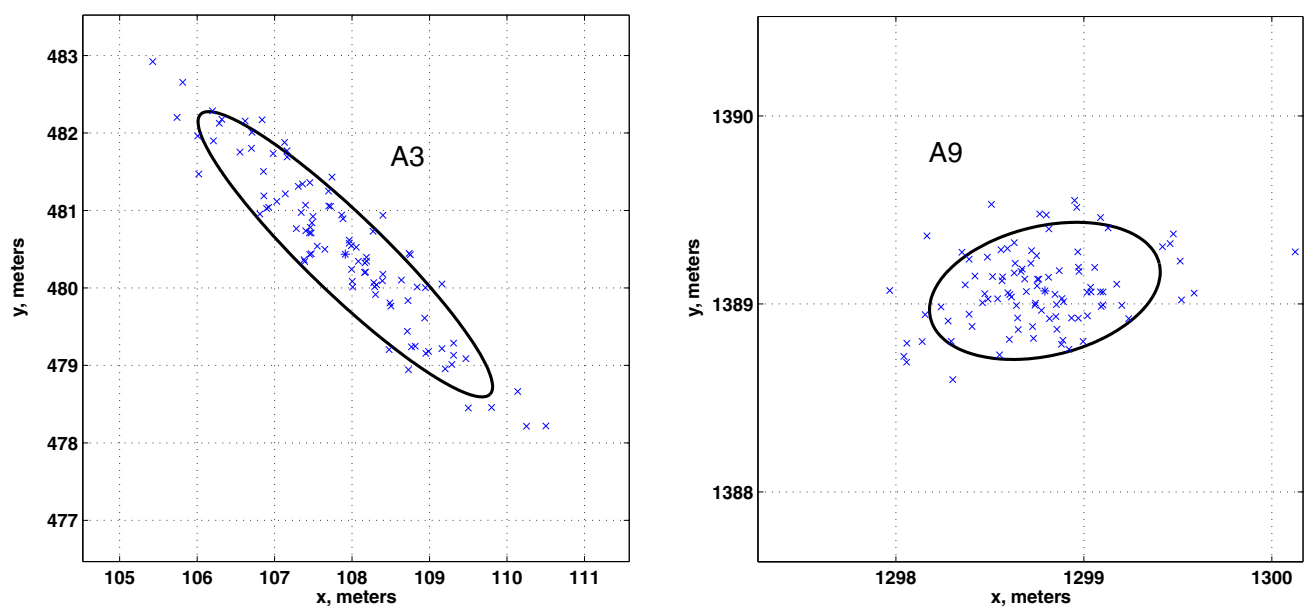

Figure 2.5: Two standard deviation location uncertainty ellipses for sensors $A 3$ and $A 9$ from Figure 2.4. 
The results of the maximum likelihood estimation procedure are also shown in Figure 2.5. The ' $x$ ' marks show the ML location estimates from 100 Monte-Carlo experiments in which randomly-generated DOA and TOA measurements were generated. The DOA and TOA measurement errors were drawn from Gaussian distributions with zero mean and variances of $\sigma_{t}=1 \mathrm{msec}$ and $\sigma_{\theta}=3^{\circ}$, respectively. The solid ellipse shows the $2 \sigma$ uncertainty region as predicted by the $\mathrm{CRB}$. We find strong agreement between the $\mathrm{CRB}$ uncertainty predictions and the Monte-Carlo experiments, which demonstrates the statistical efficiency of the ML estimator for this level of measurement uncertainty.

Figure 2.6 shows a similar uncertainty plot, but in this case we assume that both the location and orientation of sensor $A 1$ is known. In comparison with Figure 2.4, we see much larger uncertainty ellipses for the sensors, especially in the direction tangent to circles with center at sensor $A 1$. The high tangential uncertainty is primarily due to the DOA measurement uncertainty with respect to a known orientation of sensor $A 1$. By comparing Figures 2.4 and 2.6, we see that it is more desirable to know the locations of two sensors than to know the location and orientation of a single sensor; thus, equipping two sensors with GPS systems results in lower uncertainty than equipping one sensor with a GPS and a compass. In the example shown, we arbitrarily chose sensors A1 and A2 to have known locations, and they just happened to be relatively close to each other; however, choosing the two sensors with known locations to be well-separated tends to result in lower location uncertainties for the remaining sensors.

We use as a quantitative measure of performance the $2 \sigma$ uncertainty radius, defined as the radius of a circle whose area is the same as the area of the $2 \sigma$ location uncertainty ellipse. The $2 \sigma$ uncertainty radius for each sensor or source is computed as the geometric mean of the major and minor axis lengths of the $2 \sigma$ uncertainty ellipse. We find that the 


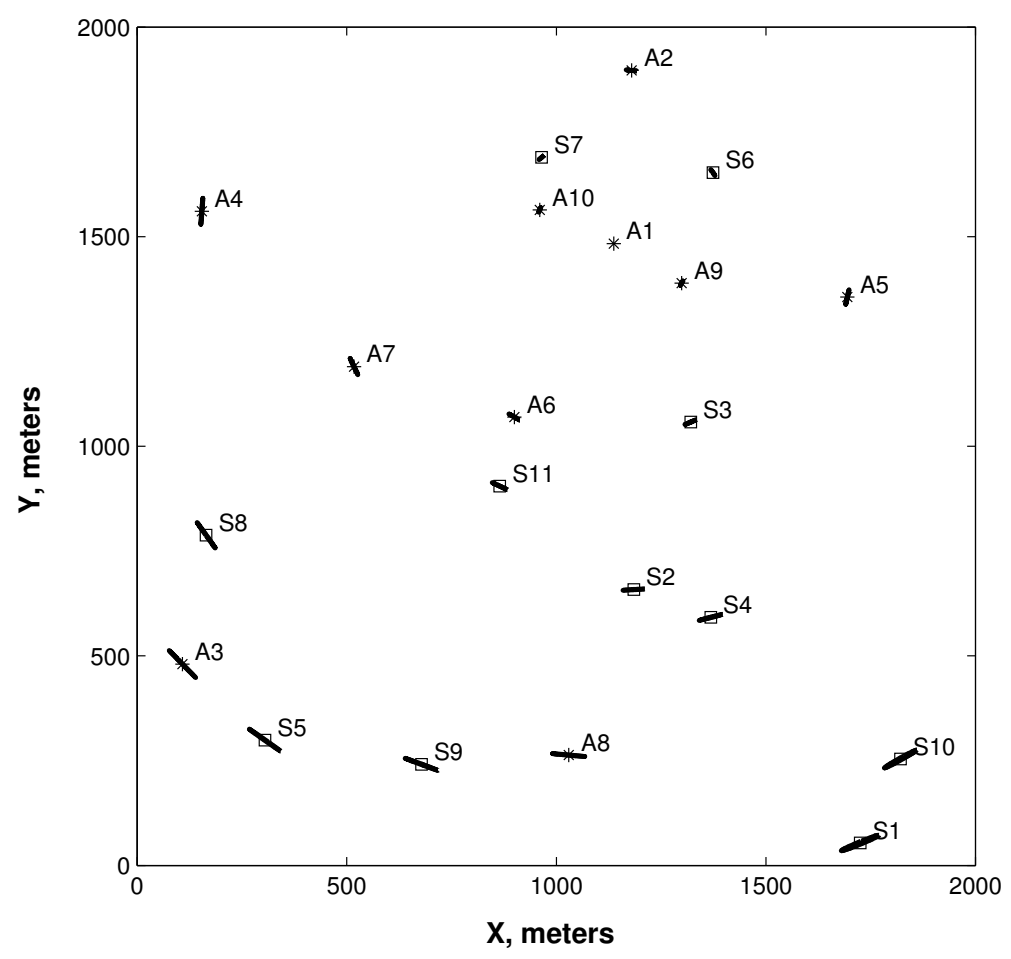

Figure 2.6: The $2 \sigma$ location uncertainty ellipses for the scene in Figure 2.4 when the location and orientation of sensor $A 1$ is assumed to be known. 
average $2 \sigma$ uncertainty radius for all ten sensors is $0.80 \mathrm{~m}$ for the example in Figure 2.4 and it is $3.28 \mathrm{~m}$ for the example in Figure 2.6.

Figure 2.7 shows the effect of increasing the number of sources on the average $2 \sigma$ uncertainty radius. We plot the average of the ten sensor $2 \sigma$ uncertainty radii, computed from the CRB, using from 2 through 11 sources, starting initially with sources $S 1$ and $S 2$ in Figure 2.4 and adding sources $S 3, S 4, \ldots, S 11$ at each step. The solid line gives the average $2 \sigma$ uncertainty radius values when sensors A1 and A2 have known locations, and the dotted line corresponds to the case when A1 has known location and orientation. The uncertainty reduces dramatically when the number of sources increases from 2 to 3 and then decreases more gradually as more sources are added.

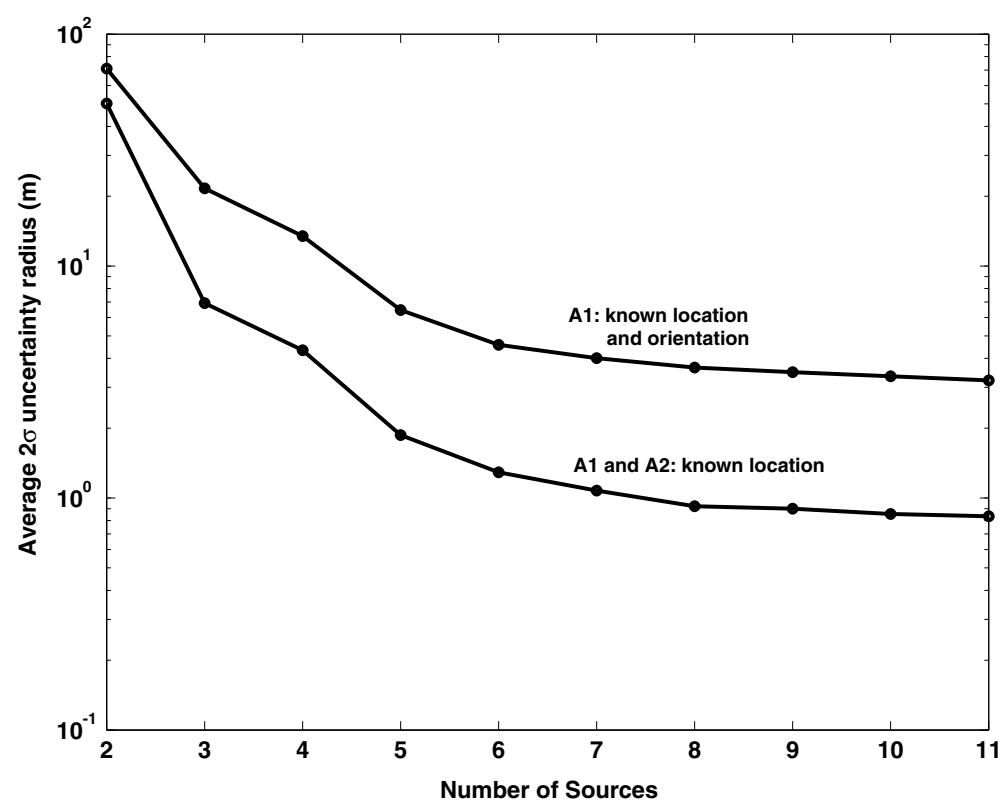

Figure 2.7: Average $2 \sigma$ location uncertainty radius for the scenes in Figures 2.4 and 2.6 as a function of the number of source signals used. 


\section{Partial Measurements}

Next, we consider the case when not all sensors detect all sources. For a sensor that is a distance $r$ from a source, we model the detection probability as

$$
P_{D}(r)=\exp ^{-\left(r / r_{0}\right)^{2}}
$$

where $r_{0}$ is a constant that adjusts the decay rate on the detection probability $\left(r_{0}\right.$ is the range

in meters at which $P_{D}=e^{-1}$ ). We assume that when a sensor detects a source, it measures both the DOA and TOA of that source.

Three detection probability profiles are considered, as shown in Figure 2.8, and correspond to $r_{0}=800 \mathrm{~m}, r_{0}=2000 \mathrm{~m}$, and $r_{0}=\infty$. Figure 2.9 shows the average $2 \sigma$ uncertainty radius values, computed from the inverse of the FIM in equation (2.20), for each of these choices of $r_{0}$. In this experiment we assume the locations of sensors A1 and A2 are known. The average number of sources detected by each sensor is also shown. For $r_{0}=2000 \mathrm{~m}$ we see only a slight uncertainty increase over the case where all sensors detect all sources. When $r_{0}=800 \mathrm{~m}$ the average location uncertainty is substantially larger because the effective number of sources seen by each sensor is small. This behavior is consistent with the average number of sources detected by each sensor, shown in Figure 2.10. For a denser set of sensors or sources, the uncertainty reduces to a value much closer to the case of full signal detection; for example, with 30 sensors and 30 sources in this region the average uncertainty is less than $1 \mathrm{~m}$ even when $r_{0}=800 \mathrm{~m}$.

\subsubsection{Field Test Results}

We present the results of applying the auto-calibration procedure to an acoustic source calibration data collection conducted during the DUNES test at Spesutie Island, Aberdeen Proving Ground, Maryland, in September 1999. In this test, four acoustic sensors are 


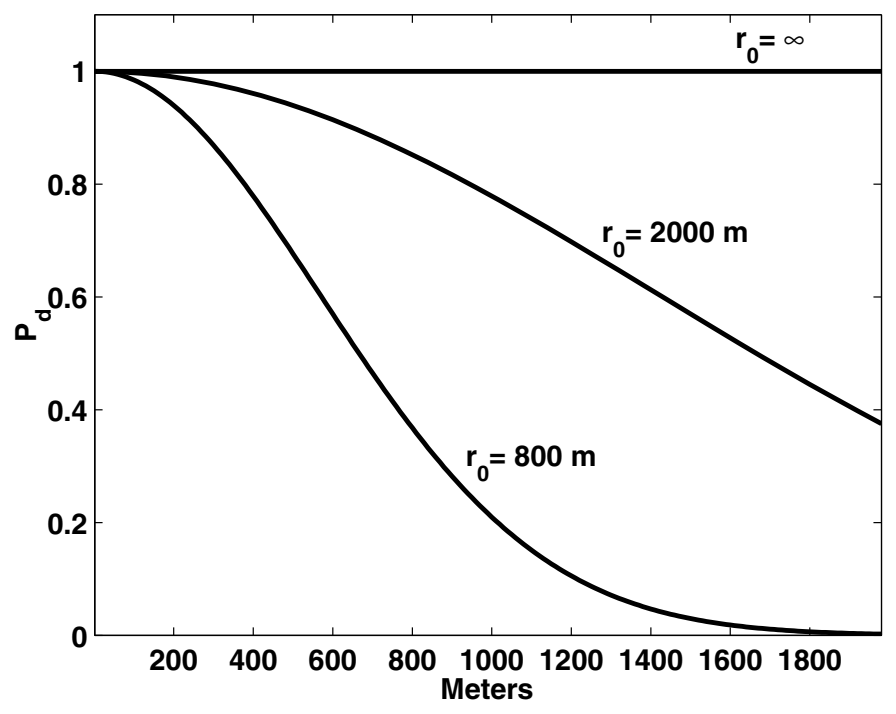

Figure 2.8: Detection probability of a source a distance $r$ from a sensor, for three values of $r_{0}$.

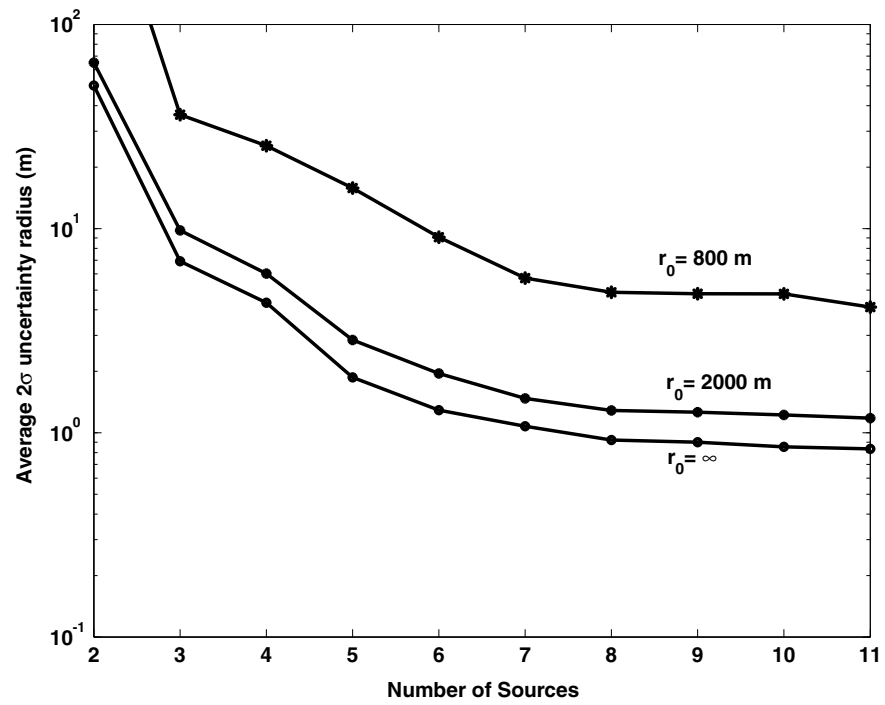

Figure 2.9: Average $2 \sigma$ location uncertainty for sensors in Figure 2.4 for three detection probability profiles. 


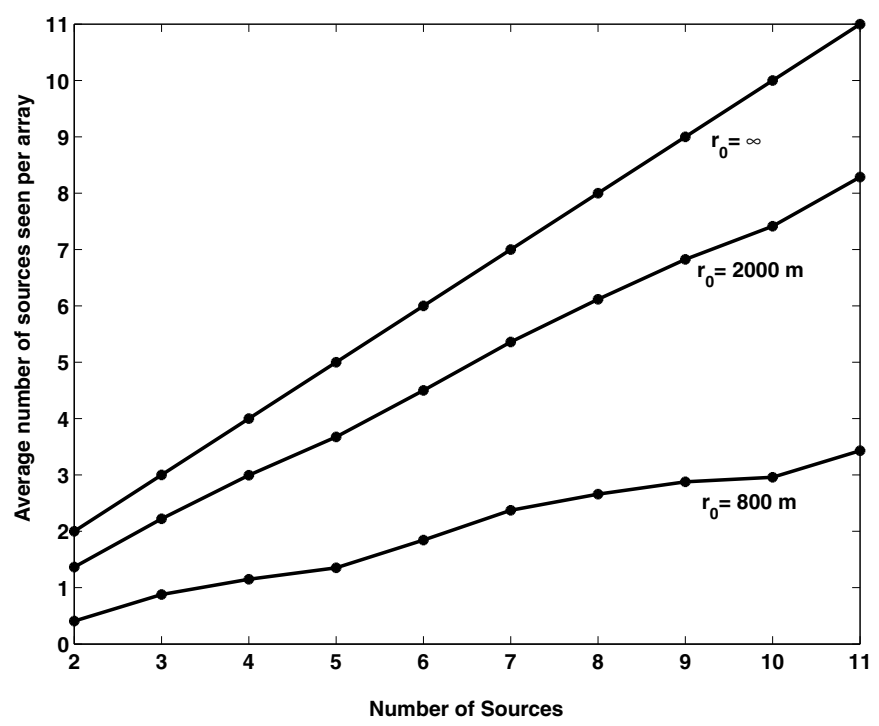

Figure 2.10: Average number of sources detected by each sensor for sensors in Figure 2.4 for three detection probability profiles.

placed at known locations 60-100 $\mathrm{m}$ apart as shown in Figure 2.11. Four acoustic source signals are also used; while exact ground truth locations of the sources are not known, it is known that each source is within approximately $1 \mathrm{~m}$ of a sensor. Each source signal is a series of bursts in the $40-160 \mathrm{~Hz}$ frequency band. Time-aligned samples of the sensor microphone signals are acquired at a sampling rate of $1005.53 \mathrm{~Hz}$. Times of arrival are estimated by cross-correlating the measured microphone signals with the known source waveform and finding the peak of the correlation function. Only a single microphone signal is available at each sensor, so while TOA measurements are obtained, no DOA measurements are available.

Figure 2.11 shows the ML estimates of sensor and source locations assuming sensor A1 has known location and the angle from A1 to A4 is known, but assuming no information about the source locations nor emission times. Because no DOA estimates are available 
from the sensors, the estimate shown in Figure 2.11 and its mirror image have identical likelihoods; we have shown only the 'correct' estimate in the figure. If we assume that the angle from A1 to A2 is known, the location errors of sensors A2, A3, and A4, are $0.14 \mathrm{~m}$, $0.40 \mathrm{~m}$, and $1.24 \mathrm{~m}$, respectively, for an average error of $0.60 \mathrm{~m}$. If we assume that the angle from $\mathrm{A} 1$ to $\mathrm{A} 3$ is known, the location errors of sensors $\mathrm{A} 2, \mathrm{~A} 3$, and $\mathrm{A} 4$, are $0.17 \mathrm{~m}$, $0.39 \mathrm{~m}$, and $1.37 \mathrm{~m}$, respectively, for an average error of $0.64 \mathrm{~m}$. Lastly, if we assume that the angle from A1 to A4 is known, the location errors of sensors A2, A3, and A4, are $0.87 \mathrm{~m}, 1.03 \mathrm{~m}$, and $0.27 \mathrm{~m}$, respectively, for an average error of $0.72 \mathrm{~m}$. The combined average error for each of these three cases is $0.65 \mathrm{~m}$. If we find the angle of rotation that minimizes the average error, we get that the location errors of sensors $\mathrm{A} 2, \mathrm{~A} 3$, and $\mathrm{A} 4$, are $0.43 \mathrm{~m}, 0.63 \mathrm{~m}$, and $0.69 \mathrm{~m}$, respectively, for an average error of $0.59 \mathrm{~m}$. In addition, the source location estimates are within $2 \mathrm{~m}$ of the sensor locations in all cases considered, consistent with our ground truth information.

Finally, we note that the calibration procedure requires low sensor communication and has reasonable computational cost. The algorithms require low communication overhead as each sensor needs to communicate only two scalar values to the CIP for each source signal it detects. Computation of the calibration solution takes place at the CIP. For the synthetic examples presented the calibration computation takes on the order of 10 seconds using Matlab on a standard personal computer. For the field test data, computation time was less than 1 second.

\subsection{Conclusions}

We have presented a procedure for calibrating the locations and orientations of a network of sensors. The calibration procedure uses source signals that are placed in the scene 


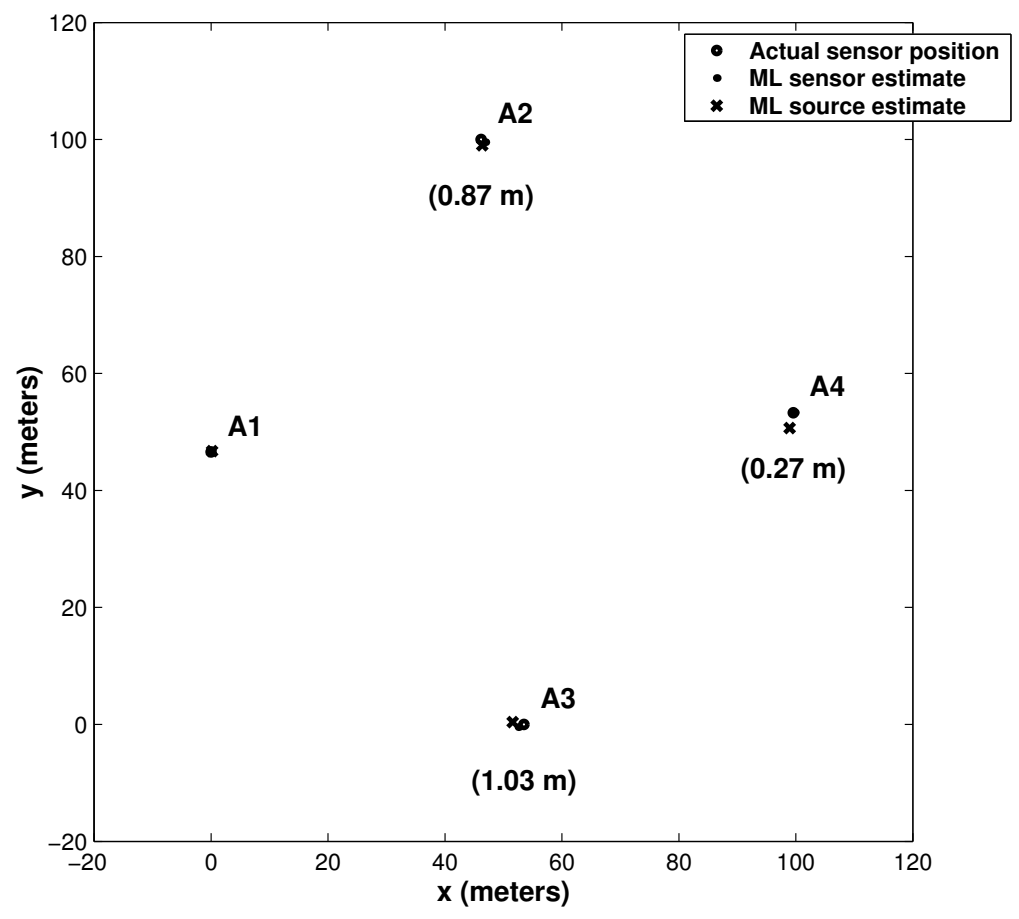

Figure 2.11: Actual and estimated sensor locations, and estimated source locations, using field test data. Sensor A1 is assumed to have known location, and the angle from sensor A1 to sensor A4 is assumed known. Error distances between actual and estimated sensor locations are shown in parentheses. 
and computes sensor and source unknowns from estimated time-of-arrival and/or directionof-arrival estimates obtained for each source-sensor pair. We present maximum likelihood solutions to four variations on this problem, depending on whether the source locations and signal emission times are known or unknown. We also discuss the existence and uniqueness of solutions and algorithms for initializing the nonlinear minimization step in the maximum likelihood estimation. A maximum likelihood calibration algorithm for the case of partial calibration measurements was also developed.

An analytical expression for the Cramér-Rao lower bound on sensor location and orientation error covariance matrix is also presented. The Cramér-Rao bound is a useful tool to investigate the effects of sensor density and source detection ranges on the self-localization uncertainty.

The algorithms require minimal communications from the sensors to a Central Information Processor, and computation of the calibration solution takes on the order of ten seconds using Matlab on a standard personal computer for examples considered. 


\section{CHAPTER 3}

\section{SELF-CALIBRATION OF SENSOR NETWORKS}

\subsection{Introduction}

Collaborative sensing has becoming increasingly important in many military and civilian applications $[1,2,3,4]$. The basic concept is to deploy a large number of low-cost, self-powered sensor nodes that acquire and process data. Typical sensors may include one or more acoustic microphones as well as seismic, magnetic, or imaging sensing capability. The collaborative sensing goals are to detect, track, and classify objects in the environment. An RF communication network links the sensors to one another and to a Central Information Processor, which in turn communicates with a higher-level information processing center (see Figure 3.1).

In order to fuse information acquired by individual sensors, one generally needs to know the location and orientation of each sensor. However, accurate prior knowledge of sensor locations and orientations is often not available. These sensors are placed in the field by persons, by an air drop, or by munition launch, and in each case the location and orientation of the placed sensor has high uncertainty. One could equip every sensor with a GPS and compass to obtain location and orientation information, but this adds to the expense and power requirements of the sensor and increases its susceptibility to detection or 


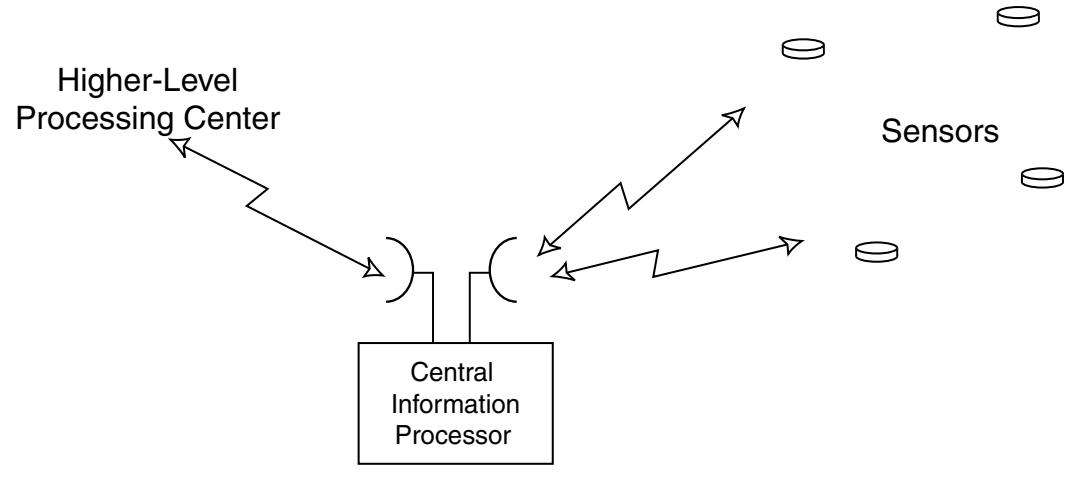

Figure 3.1: Sensor network architecture. A network of low-cost sensors are deployed in a region. Each sensor communicates to a local Central Information Processor, which relays information to a higher-level information processing center.

jamming. Thus, there is interest in developing methods to self-localize the sensor network with a minimum of additional hardware, processing, or communication.

Self-localization in sensor networks is an active area of current research $[17,27,12]$. Savvides et al. consider iterative multilateration-based techniques [18], and Bulusu et al. consider low-cost localization methods that employ beacon signals at known locations [12, 19]. Cevher and McClellan consider sensor network self-calibration using a single acoustic source that travels along a straight line [14]. Bearings-only localization methods have also been considered [16].

Many self-calibration techniques require knowledge of the absolute locations of some nodes. In particular, the above techniques assume exact knowledge of a small number of "anchor nodes". Such information is often not available, or is costly to provide. In this chapter we consider an approach to sensor network self-calibration using a combination of calibration sources and uncertain prior location information. The proposed self-calibration approach entails placing a number of signal sources in the same region as the sensors (see Figure 3.2). Neither the source locations nor their signal emission times are assumed to 
be known. Each source generates a signal that is detected by some of the sensors, and each sensor measures the time-of-arrival (TOA) and, if possible, direction-of-arrival (DOA) of the source signal. These measurements permit estimating the relative locations and orientations of the sensors.

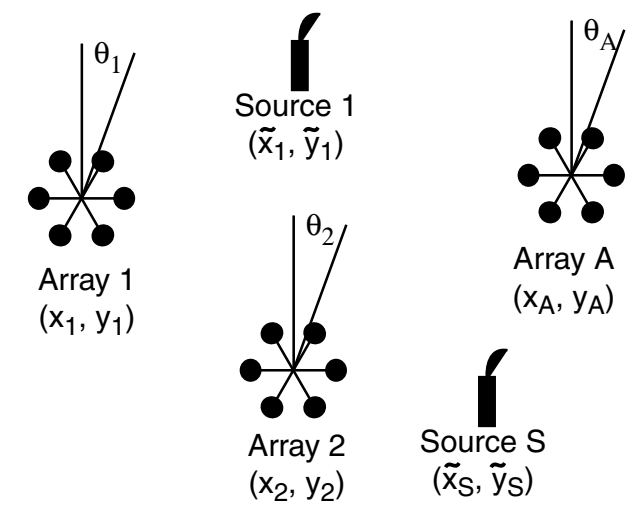

Figure 3.2: Sensor self-localization scenario. Sensors are placed in the field at unknown locations and orientations. Calibrations sources, also at unknown locations, generate signals time-of-arrival and direction-of-arrival measurements at the sensors are used for network self-calibration.

In addition, we assume uncertain prior location information for some of the sensors. Uncertain prior information about sensor location may be available in practice from aimpoints of munition-deployed sensors, or from aircraft location at the point of an air drop of a sensor. Although the aimpoints may be known, the actual sensor location typically has high uncertainty (on the order of tens of meters) from this aimpoint.

We develop maximum a posteriori (MAP) estimates of the absolute locations and orientations of the sensors from the prior location information along with the source-to-sensor TOA and DOA estimates. We also derive bounds on localization accuracy. We parameterize the localization problem as a sum of two parts: an absolute location and orientation of 
the entire sensor network, and the relative location and orientation of each sensor with respect to this absolute location. We compute the Cramér-Rao bound for the relative location parameters and derive the uncertainty of the MAP absolute location estimate. The relative location errors are typically (much) smaller than the prior location uncertainties.

Finally, we present experimental results on both synthetic data and field measurements to demonstrate the effectiveness of the proposed techniques.

\subsection{Self-Localization Problem Formulation}

Assume we have a set of $A$ sensors in a plane, each with unknown location $\left\{a_{i}=\right.$ $\left.\left(x_{i}, y_{i}\right)\right\}_{i=1}^{A}$ and unknown orientation angle $\theta_{i}$ with respect to a reference direction $(e . g$, North); see Figure 3.2. We consider the two-dimensional problem in which the sensors lie in a plane and the unknown reference direction is azimuth; an extension to the threedimensional case is possible using similar techniques.

In the sensor field are also placed $S$ point calibration source signals at locations $\left\{s_{j}=\right.$ $\left.\left(\tilde{x}_{j}, \tilde{y}_{j}\right)\right\}_{j=1}^{S}$. The source locations are in general unknown. Each source emits a finitelength signal that begins at time $t_{j}$; the emission times are also in general unknown.

Define the parameter vector $\alpha$ containing the sensor and source unknown parameters:

$$
\begin{aligned}
\alpha & =\left[\beta^{T}, \gamma^{T}\right]^{T} \quad(3(A+S) \times 1) \\
\beta & =\left[x_{1}, y_{1}, \theta_{1}, \ldots, x_{A}, y_{A}, \theta_{A}\right]^{T} \quad(3 A \times 1) \\
\gamma & =\left[\tilde{x}_{1}, \tilde{y}_{1}, t_{1}, \ldots, \tilde{x}_{S}, \tilde{y}_{S}, t_{S}\right]^{T} \quad(3 S \times 1)
\end{aligned}
$$

Here, $\beta$ contains the sensor unknown parameters and $\gamma$ contains the unknown nuisance source signal parameters. Let $n_{\alpha}=3(A+S)$ denote the length of $\alpha$.

We assume that some prior information about $\alpha$ is available. Typically the prior information encodes the nominal locations and uncertainty of some of the sensors or sources. 
We express this this prior information probabilistically as a prior probability density function $f_{0}(\alpha)$. As an example, one can assume $f(\alpha)$ is Gaussian distributed with known mean and covariance, so

$$
f_{0}(\alpha)=\mathcal{N}\left(\alpha_{0}, \Sigma_{0}\right)
$$

where $\alpha_{0}$ and $\Sigma_{0}$ are given.

\subsubsection{Absolute and Relative Calibration}

It is useful to express the location parameters in terms of a relative location and orientation with respect to the sensor-source centroid. To this end, define the centroid location and orientation, $\alpha_{c}(\alpha)=\left[x_{c}, y_{c}, \theta_{c}\right]^{T}$, by

$$
x_{c}=\frac{1}{A+S}\left[\sum_{i=1}^{A} x_{i}+\sum_{j=1}^{S} \tilde{x}_{j}\right] \quad y_{c}=\frac{1}{A+S}\left[\sum_{i=1}^{A} y_{i}+\sum_{j=1}^{S} \tilde{y}_{j}\right] \quad \theta_{c}=\frac{1}{A} \sum_{i=1}^{A} \theta_{i}
$$

We see that $\alpha_{c}=B \alpha$ for a $\left(3 \times n_{\alpha}\right)$ matrix $B$ with elements defined in (3.5).

For any parameter vector $\alpha$, define the corresponding relative location vector $\alpha_{r}$ by transforming the points in $\alpha$ such that $B \alpha_{r}=0$. The transformation is defined as follows for a vector $\alpha$ with centroid $\alpha_{c}=\left[x_{c}, y_{c}, \theta_{c}\right]^{T}$. For the $i$ th sensor with parameters $\left(x_{i}, y_{i}, \theta_{i}\right)$, which are the $3(i-1)+1$ st to $3(i-1)+3 r d$ elements of $\alpha$, the corresponding elements of $\alpha_{r}$ are given by

$$
\left[\begin{array}{c}
x_{i, r} \\
y_{i, r} \\
\theta_{i, r}
\end{array}\right]=\left[\begin{array}{ccc}
\cos \theta_{c} & \sin \theta_{c} & 0 \\
-\sin \theta_{c} & \cos \theta_{c} & 0 \\
0 & 0 & 1
\end{array}\right]\left[\begin{array}{c}
x_{i}-x_{c} \\
y_{i}-y_{c} \\
\theta_{i}-\theta_{c}
\end{array}\right]
$$

Similarly, for the $j$ th source with parameters $\left(\tilde{x}_{j}, \tilde{y}_{j}, t_{j}\right)$ (which are the $3(A+j-1)+1 s t$ to $3(A+j-1)+3 r d$ elements of $\alpha$, the corresponding elements of $\alpha_{r}$ are given by

$$
\left[\begin{array}{c}
\tilde{x}_{j, r} \\
\tilde{y}_{j, r} \\
t_{j, r}
\end{array}\right]=\left[\begin{array}{ccc}
\cos \theta_{c} & \sin \theta_{c} & 0 \\
-\sin \theta_{c} & \cos \theta_{c} & 0 \\
0 & 0 & 1
\end{array}\right]\left[\begin{array}{c}
\tilde{x}_{j}-x_{c} \\
\tilde{y}_{j}-y_{c} \\
t_{j}
\end{array}\right]
$$


From the above definitions, it is clear that there is a one-to-one relationship between a parameter vector $\alpha$ and the two vectors $\alpha_{r}$ and $\alpha_{c}$. Note that $t_{j}$ remains unchanged by the transformation. The inverse transformations are readily obtained from (3.6)-(3.7). We remark that even though the centroid $\alpha_{c}$ can be computed linearly $\left(\alpha_{c}=B \alpha\right)$, the transformation of a vector $\alpha$ to obtain $\alpha_{r}$ is nonlinear because all locations have to be rotated by $\theta_{c}$. We will use $\alpha$ and $\left(\alpha_{r}, \alpha_{c}\right)$ interchangeably in the remainder of the paper.

\subsubsection{Calibration Measurements}

Each emitted source signal is detected by all of the sensors in the field, and each sensor measures the time-of-arrival and direction-of-arrival for that source. We denote the measured TOA at sensor $i$ of source $j$ as $t_{i j}$ and the measured DOA as $\theta_{i j}$. The DOA measurements are made with respect to a frame of reference local to the sensor. The times of arrival are measured with respect to a known, common time base. The time base can be established, for example, by using them RF communication network linking the sensors. The time base needs to be accurate to on the order of the sensor's time of arrival measurement uncertainty (which is $1 \mathrm{msec}$ in the examples considered in Section 6).

The set of $2 A S$ calibration measurements are gathered in a vector

$$
X=\left[\begin{array}{c}
\operatorname{vec}(T) \\
\operatorname{vec}(\Theta)
\end{array}\right]^{T} \quad(2 A S \times 1)
$$

where $\operatorname{vec}(M)$ stacks the elements of a matrix $M$ columnwise and where

$$
T=\left[\begin{array}{cccc}
t_{11} & t_{12} & \ldots & t_{1 S} \\
t_{21} & t_{22} & \ldots & t_{2 S} \\
\vdots & \vdots & \ddots & \vdots \\
t_{A 1} & t_{A 2} & \ldots & t_{A S}
\end{array}\right], \quad \Theta=\left[\begin{array}{cccc}
\theta_{11} & \theta_{12} & \ldots & \theta_{1 S} \\
\theta_{21} & \theta_{22} & \ldots & \theta_{2 S} \\
\vdots & \vdots & \ddots & \vdots \\
\theta_{A 1} & \theta_{A 2} & \ldots & \theta_{A S}
\end{array}\right]
$$

Each sensor transmits its $2 S$ noisy TOA and DOA measurements to a Central Information Processor (CIP), and these $2 A S$ measurements, along with the measurement uncertainty 
and prior uncertainty $f_{0}(\alpha)$ form the information with which the CIP computes the sensor calibration. The communication cost to the CIP is low, and the calibration processing can be entirely performed by the CIP.

We denote the true TOA and DOA of source signal $j$ at sensor $i$ as $\tau_{i j}(\alpha)$ and $\phi_{i j}(\alpha)$, respectively, and include their dependence on the parameter vector $\alpha$; they are given by:

$$
\begin{aligned}
\tau_{i j}(\alpha) & =t_{j}+\left\|a_{i}-s_{j}\right\| / c \\
\phi_{i j}(\alpha) & =\theta_{i}+\angle\left(a_{i}, s_{j}\right)
\end{aligned}
$$

where $a_{i}=\left[x_{i}, y_{i}\right]^{T}, a_{j}=\left[\tilde{x}_{j}, \tilde{y}_{j}\right]^{T},\|\cdot\|$ is the Euclidean norm, $\angle(\xi, \eta)$ is the angle between the points $\xi, \eta \in \mathcal{R}^{2}$, and $c$ is the signal propagation velocity.

Each element of $X$ has measurement uncertainty; we model the uncertainty as

$$
X=\mu(\alpha)+E
$$

where $\mu(\alpha)$ is the noiseless measurement vector whose elements are given by equations (3.10) and (3.11) for values of $i, j$ that correspond to the vector stacking operation in (3.8), and where $E$ is a random vector with known probability density function.

The self-calibration problem, then, is: given the measurement $X$, estimate $\beta$. The parameters in $\gamma$ are in general unknown and are nuisance parameters that may also need to be estimated.

Significantly, the measurements in $X$ provide information about the relative sensor and source locations but not about the centroid. Thus, $X$ enables us to estimate $\alpha_{r}$ but provides no information about $\alpha_{c}$. 


\subsection{Bayesian Self-Calibration}

We consider $\alpha$ as a random vector with prior probability density function (pdf) $f_{0}(\alpha)$. The measurement vector informs us about $\alpha$ as quantified by the posterior probability $f(\alpha \mid X)$. From Bayes' rule,

$$
f(\alpha \mid X)=\frac{f(X \mid \alpha) f_{0}(\alpha)}{f(X)}
$$

We choose as our estimate maximum a posteriori (MAP) estimate, which is the value of $\alpha$ that maximizes the posterior probability density of $\alpha$ :

$$
\hat{\alpha}=\arg \max _{\alpha} f(\alpha \mid X)=\arg \max _{\alpha} f(X \mid \alpha) f_{0}(\alpha)
$$

Note that the measurements $X$ are independent of absolute location; thus, $f(X \mid \alpha)=$ $f\left(X \mid \alpha_{r}\right)$. Combining this fact with the negative logarithm of equation (3.14) yields

$$
\hat{\alpha}=\arg \min _{\left(\alpha_{r}, \alpha_{c}\right)}\left[-\ln f\left(X \mid \alpha_{r}\right)-\ln f_{0}(\alpha)\right]
$$

where $\alpha_{c}=\left[x_{c}, y_{c}, \theta_{c}\right]^{T}$ is a $3 \times 1$ centroid vector.

\subsubsection{Gaussian Measurement Uncertainty}

If we assume the measurement uncertainty $E$ in equation (3.12) is Gaussian with zero mean and known covariance $\Sigma_{X}$, then

$$
\begin{aligned}
f(X ; \alpha) & =\frac{1}{(2 \pi)^{A S}\left|\Sigma_{X}\right|^{1 / 2}} \exp \left\{-\frac{1}{2} Q(X ; \alpha)\right\} \\
Q(X ; \alpha) & =[X-\mu(\alpha)]^{T} \Sigma_{X}^{-1}[X-\mu(\alpha)]
\end{aligned}
$$

A special case is when the measurement errors are uncorrelated and the TOA and DOA measurement errors have variances $\sigma_{t}^{2}$ and $\sigma_{\theta}^{2}$, respectively; equation (3.17) then becomes

$$
Q(X ; \alpha)=\sum_{i=1}^{A} \sum_{j=1}^{S}\left[\frac{\left(t_{i j}-\tau_{i j}(\alpha)\right)^{2}}{\sigma_{t}^{2}}+\frac{\left(\theta_{i j}-\phi_{i j}(\alpha)\right)^{2}}{\sigma_{\theta}^{2}}\right]
$$


If we further assume that the prior information is Gaussian with mean $\alpha_{0}$ and covariance $\Sigma_{0}$, then

$$
f_{0}(\alpha)=\frac{1}{(2 \pi)^{3(A+S) / 2}\left|\Sigma_{0}\right|^{1 / 2}} \exp \left\{-\frac{1}{2}\left[\alpha-\alpha_{0}\right]^{T} \Sigma_{0}^{-1}\left[\alpha-\alpha_{0}\right]\right\}
$$

Note that some elements of $\alpha$ may have no prior information, in which case the above pdf will be reduced in dimension accordingly.

For the case of Gaussian priors and Gaussian measurement errors, equation (3.15) yields

$$
\hat{\alpha}=\arg \min _{\left(\alpha_{r}, \alpha_{c}\right)}[X-\mu(\alpha)]^{T} \Sigma_{X}^{-1}[X-\mu(\alpha)]+\left[\alpha-\alpha_{0}\right]^{T} \Sigma_{0}^{-1}\left[\alpha-\alpha_{0}\right]
$$

Equations (3.15) and (3.20) both involve minimization of a nonlinear function of $\alpha$; for the Gaussian case, (3.20) yields a nonlinear least squares minimization problem. In the next section we discuss various approaches to solve this minimization problem.

\subsection{Algorithms for Self-Calibration}

First, we write (3.15) as

$$
\hat{\alpha}=\arg \min _{(\alpha, c)}\left[J_{1}\left(\alpha_{r}\right)+J_{2}\left(\alpha_{r}, c\right)\right]
$$

where $J_{1}\left(\alpha_{r}\right)=-\ln f\left(X \mid \alpha_{r}\right)$ and $J_{2}\left(\alpha_{r}, c\right)=-\ln f_{0}(\alpha)$.

In developing algorithms for minimizing (3.21), it is useful to consider separately the case for which some prior information on $\alpha$ is known with relatively high accuracy and the case for which the prior information has high uncertainty. For the former case, one can use the high-accuracy prior information to efficiently determine an initial $\alpha$ to form the starting point for the nonlinear minimization. For example, if we know the prior location of at least two sensors with high accuracy, then the techniques developed in Moses et al. can be used to initialize the locations orientations, and emission times of the sources and sensors [28]. 
The more difficult case is the one for which the prior location information has high uncertainty. In this case, we form an initial estimate by making the following approximation: we assume that $\alpha_{r}$ that minimizes (3.21) is approximately equal to $\hat{\alpha}_{r}$, where

$$
\hat{\alpha}_{r}=\arg \min _{\left(\alpha_{r}\right)} J_{1}\left(\alpha_{r}\right)
$$

Next, consider the minimization of $J_{1}\left(\alpha_{r}\right)$. We proceed as follows. First, note that there is a one-to-one correspondence between a parameter vector $\alpha_{r}$ and a parameter vector $\alpha_{1}$ for which $x_{1}=y_{1}=\theta_{1}=0$. This is not surprising, since in both cases there are three degrees of freedom removed from a parameter vector $\alpha$. One can compute $\alpha_{r}$ from $\alpha_{1}$ using equations similar to (3.6)-(3.7). Thus, one method for finding $\hat{\alpha}_{r}$ is to find

$$
\hat{\alpha}_{1}=\arg \min _{\alpha_{1}} J_{1}\left(\alpha_{1}\right)
$$

where $\alpha_{1}$ satisfies $x_{1}=y_{1}=\theta_{1}=0$, and then to convert $\hat{\alpha}_{1}$ to a corresponding $\hat{\alpha}_{r}$ using this one-to-one transformation. An algorithm for finding $\hat{\alpha}_{1}$ in (3.23) is given in Moses et al. [28].

Once $\hat{\alpha}_{r}$ is obtained from (3.22), finding $\hat{\alpha}$ that solves (3.21) remains. This generally involves a nonlinear minimization over the parameters in $\alpha$. Initial estimates for $\alpha_{r}$ have already been computed, so we need to initialize the $3 \times 1$ constraint vector $\alpha_{c}$. One way to initialize $\alpha_{c}$ is to find the translation components $x_{c}$ and $y_{c}$ to align the centroid of the sensor and source locations to centroid of the prior location information. For example, if the prior information $f_{0}(\alpha)$ is Gaussian with mean $\alpha_{0}$, and covariance $\Sigma_{0}=k I$ for some constant $k$, one finds that the ML estimate of the centroid given $\alpha_{r}$ is found by setting $\left(x_{c}, y_{c}\right)$ to the centroid of the known sensor and source locations given in $\alpha_{0}$. In practice, it may be that prior location information for only a subset of sensors or sources is known; in this case one would set the centroids equal only on the subset for which prior information 
is known. The remaining centroid parameter, $\theta_{c}$, can be initialized by a coarse brute-force search on the interval $[0,2 \pi)$. For Gaussian prior locations with general $\Sigma_{0}$, the solution for $x_{c}$ and $y_{c}$ that minimizes (3.21) can be found in closed form as the solution to a $2 \times 2$ matrix equation.

Once an initial estimate of $\alpha_{c}$ is found, one can find $\hat{\alpha}$ from (3.21) using an iterative descent procedure. In our implementation we used the Matlab function lsqnonlin.

If the prior knowledge has high uncertainty, an approximate solution to (3.21), with lower computational cost, may be found as follows. If the prior knowledge has high uncertainty, then $J_{2}$ depends only weakly on $\alpha_{r}$, so the optimal $\alpha_{r}$ vector is approximately given by the solution to (3.22). Then, we can solve for the remaining three parameters in $\alpha_{c}$ by inserting $\hat{\alpha}_{r}$ into equation (3.21) and finding $\hat{\alpha}_{c}$; that is:

$$
\hat{\alpha}_{c}=\arg \min _{\alpha_{c}}\left[J_{1}\left(\hat{\alpha}_{r}\right)+J_{2}\left(\hat{\alpha}_{r}, c\right)\right]
$$

As discussed above, if the prior uncertainty model is Gaussian, the translation parameters $x_{c}$ and $y_{c}$ can be found in closed form, and the minimization in (3.24) reduces to a search over the scalar parameter $\theta_{c}$.

\subsubsection{Uncertainty in Propagation Velocity}

Thus far we have assumed that the signal propagation velocity, $c$, is known. In this section we discuss modifications to the above approach when there is some uncertainty in $c$.

First, we note that the TOA and DOA measurements provide no information for estimation of $c$. Uncertainty in $c$ results in an uncertainty on the scaling of the overall sourcesensor locations in $\alpha_{r}$. Thus, if $c$ is unknown, we append $c$ to the set of unknowns in $\alpha_{c}$. 
For the case of Gaussian prior information with uncorrelated and equal variance uncertainty in the sensor and source $x$ and $y$ locations, equation (3.24) (where now $\alpha_{c}=$ $\left[x_{c}, y_{c}, \theta_{c}, c\right]$ can be solved for in closed-form using a least-squares estimate [29]. Said another way, the conditional maximum likelihood estimate of $c$ given $\alpha_{r}$ is obtained by solving a linear set of equations. It is interesting to note that a closed-form solution is possible when $c$ is unknown but not when $c$ is known.

\subsection{Estimation Accuracy}

In this section we establish the accuracy of both the maximum likelihood estimate of $\alpha_{r}$ for the case of no prior information, and for the MAP estimate of $\alpha$ that incorporates both the TOA/DOA measurements and the prior location information. We use the Fisher Information Matrix (FIM) and the Cramér-Rao Bound (CRB) as tools to analytically determine estimation accuracy. The Cramér-Rao Bound (CRB) gives a lower bound on the covariance of any unbiased estimate of $\alpha$. It is a tight bound in the sense that $\hat{\alpha}_{M L}$ has parameter uncertainty given by the CRB for high measurement signal-to-noise ratio; that is, as $\max _{i}\left(\Sigma_{X}\right)_{i i} \rightarrow 0$. Thus, the CRB is a useful tool for analyzing calibration uncertainty. It is computed from the inverse of the Fisher Information Matrix when the inverse exists. In our case, the FIM for $\alpha_{r}$ is singular, and a pseudoinverse-based solution must be used. In the addition of prior information, we can compute the covariance of the MAP $\alpha$ estimate.

\subsubsection{Estimation Accuracy of Relative Location}

First, we establish a lower bound on the covariance of an unbiased estimator of $\alpha_{r}$ based on measurements $X$. In this derivation, we assume no prior knowledge on $\alpha$; that is, we assume $\alpha$ is a deterministic unknown parameter vector. Since $X$ provides no information about the centroid parameters, we find that the Fisher information matrix of $\alpha$ is singular; in 
fact, the null space of the Fisher information matrix generally has rank three, corresponding to the three degrees of freedom in an arbitrary translation and rotation of the sensor and source locations. However, the constraints imposed on $\alpha_{r}$ are such that its covariance estimate is nonsingular in all but degenerate cases. An example of a degenerate case is when all sensors are collinear and a calibration source is collinear with the sensors and not between any two sensors.

The CRB can be computed from the Fisher Information Matrix. The Fisher Information Matrix of $\alpha$ is given by [24]

$$
I_{\alpha}=E\left\{\left[\nabla_{\alpha} \ln f(T, \Theta ; \alpha)\right]\left[\nabla_{\alpha} \ln f(T, \Theta ; \alpha)\right]^{T}\right\}
$$

For the Gaussian estimation error case, the partial derivatives are readily computed from equation (3.16); we find that

$$
I_{\alpha}=\left[G^{\prime}\left(\alpha_{1}\right)\right]^{T} \Sigma_{X}^{-1}\left[G^{\prime}\left(\alpha_{1}\right)\right]
$$

where $G^{\prime}\left(\alpha_{1}\right)$ is the $2 A S \times n_{\alpha}$ matrix whose $i j$ th element is $\partial \mu_{i}\left(\alpha_{1}\right) / \partial\left(\alpha_{1}\right)_{j}$.

The Fisher Information Matrix is rank deficient due to the translational and rotational ambiguity in the self-calibration solution [28]. However, the CRB of $\alpha_{r}$ is finite, and given by $[30,24]$ :

$$
C_{\alpha_{r}}=P\left[I_{\alpha}\right]^{\dagger} P
$$

where $(\cdot)^{\dagger}$ denotes the Moore-Penrose pseudoinverse and where $P$ is a projection matrix given by

$$
\begin{aligned}
P & =I-M\left(M^{T} M\right)^{-1} M^{T} \\
M & =\left[\begin{array}{cccc|ccc}
M_{1} & M_{2} & \cdots & M_{A} \mid \tilde{M}_{1} & \tilde{M}_{2} & \cdots & \tilde{M}_{S}
\end{array}\right]^{T} \\
M_{i} & =\left[\begin{array}{ccc}
1 & 0 & 0 \\
0 & 1 & 0 \\
-y_{i} & x_{i} & 1
\end{array}\right], \quad \tilde{M}_{j}=\left[\begin{array}{ccc}
1 & 0 & 0 \\
0 & 1 & 0 \\
-\tilde{y}_{j} & \tilde{x}_{j} & 0
\end{array}\right]
\end{aligned}
$$




\subsubsection{Accuracy of Maximum A Posteriori Absolute Location Estimate}

When prior information is available, the covariance of the MAP estimate $\hat{\alpha}$ from (3.21) is found as follows. Since the prior information and the measurement information are independent, the information matrix associated with both is the sum of each. This total information matrix is given by [24]

$$
I_{\alpha}^{0} \geq \Sigma_{0}^{-1}+I_{\alpha}
$$

where $I_{\alpha}$ is given by equation (3.25) and where we have assumed a Gaussian prior. The covariance of the MAP estimate is bounded by the inverse of this matrix (if it exists) [24], so

$$
E\left\{[\hat{\alpha}-\alpha][\hat{\alpha}-\alpha]^{T}\right\} \geq\left[I_{\alpha}^{0}\right]^{-1}
$$

For High SNR the MAP estimator is expected to achieve this bound.

\subsection{Experimental Results}

This section presents numerical examples of the self-calibration procedure. First, we present a synthetically-generated example consisting of ten sensors and eleven sources placed randomly in a $2 \mathrm{~km} \times 2 \mathrm{~km}$ region. Second, we present results from field measurements using acoustic sources and sensors.

\subsubsection{Synthetic Data Example}

We consider a case in which ten sensors are randomly placed in a $2 \mathrm{~km} \times 2 \mathrm{~km}$ region. In addition, eleven sources are randomly placed in the same region. In both cases the nominal locations of the sensors and sources are first randomly chosen. Then, the actual locations are chosen as random Gaussian perturbations from the nominal locations, where 
the perturbation is found by adding Gaussian noise with zero mean and standard deviation of 10 meters to each location. The sensor orientations and source emission times are also randomly chosen. Figure 3.3 shows the actual locations of the sensors and sources.

Next, TOA and DOA measurements are simulated. We assume every sensor detects each source emission and measures the TOA and DOA of the source. The measurement uncertainties are Gaussian with standard deviations of $\sigma_{t}=1 \mathrm{msec}$ for the TOAs and $\sigma_{\theta}=3^{\circ}$ for the DOAs. Neither the locations nor emission times of the sources are assumed to be known.

Figure 3.3 also shows the two standard deviation $(2 \sigma)$ location uncertainty ellipses for the relative location estimates of both the sources and sensors. The ellipses are obtained from the $2 \times 2$ covariance submatrices of the $\mathrm{CRB}$ in equation (3.26) that correspond to the relative location parameters in $\alpha_{r}$ of each sensor or source. These ellipses appear as small dots in the figure; enlarged views for two sensors are also shown.

The results of the maximum likelihood estimation of relative location are also shown in Figure 3.4. The ' $x$ ' marks show the ML location estimates from 200 Monte-Carlo experiments with randomly-generated DOA and TOA measurements. The DOA and TOA measurement errors were drawn from Gaussian distributions with zero mean and variances of $\sigma_{t}=1 \mathrm{msec}$ and $\sigma_{\theta}=3^{\circ}$, respectively. The ellipse shows the 2-standard deviation $(2 \sigma)$ relative location uncertainty region as predicted from the CRB. We find good agreement between the CRB uncertainty predictions and the Monte-Carlo experiments, which demonstrates the statistical efficiency of the ML estimator for this level of measurement uncertainty.

We summarize the ten location uncertainty ellipses into a single quantity by first defining the sensor or source $2 \sigma$ uncertainty radius as the radius of a circle whose area is the 


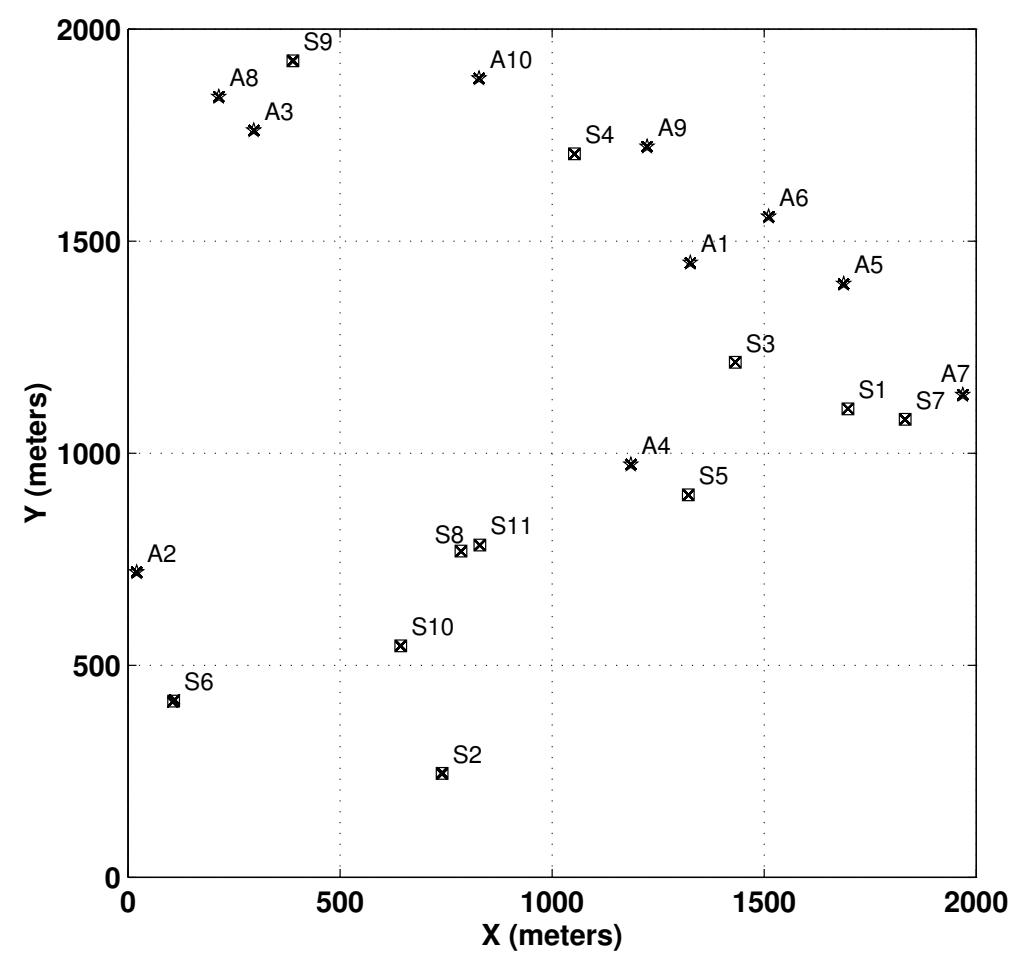

Figure 3.3: Top: Example scene showing ten sensors A1-A10 (stars) and eleven sources S1-S11 (squares). Also shown are the $2 \sigma$ location uncertainty ellipses of the sensors and sources; these are on average less than $0.5 \mathrm{~m}$ in radius and show as small dots. Bottom: enlarged views near sensors A6 and A8, showing $2 \sigma$ location uncertainty ellipses along with location estimates from 200 Monte-Carlo experiments.
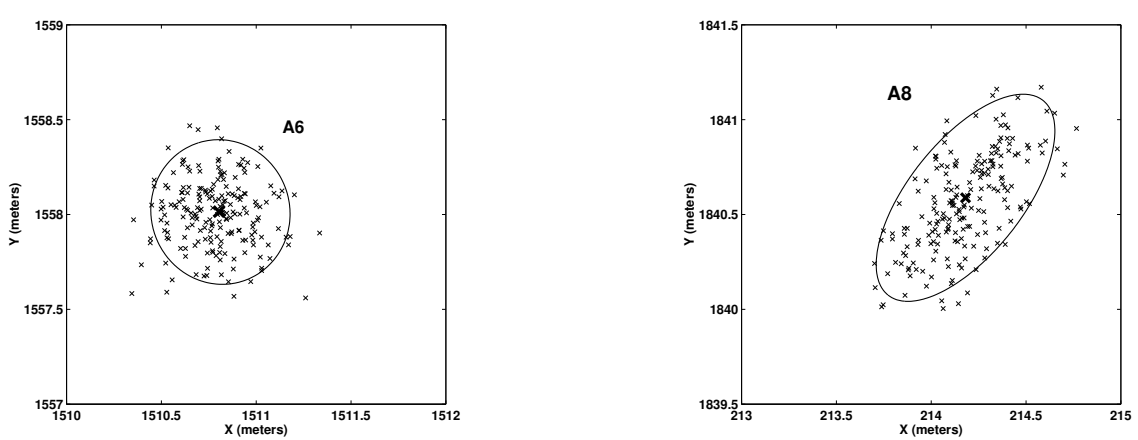

Figure 3.4: Two standard deviation location uncertainty ellipses for sensors $A 6$ and $A 8$ from Figure 3.3. 


\begin{tabular}{|c|c|c|}
\hline & $\begin{array}{c}\text { Theoretical } \\
\text { Value }\end{array}$ & $\begin{array}{c}\text { Monte-Carlo } \\
\text { Simulation }\end{array}$ \\
\hline Relative Location & $0.47 \mathrm{~m}$ & $0.43 \mathrm{~m}$ \\
\hline Absolute Location & $5.19 \mathrm{~m}$ & $5.09 \mathrm{~m}$ \\
\hline
\end{tabular}

Table 3.1: Average $2 \sigma$ uncertainty radius for relative and absolute sensor calibration for the example presented.

same as the area of the $2 \sigma$ location uncertainty ellipse. The $2 \sigma$ uncertainty radius for each sensor or source is computed as the geometric mean of the major and minor axis lengths of the $2 \sigma$ uncertainty ellipse. We then average these uncertainty radii to obtain a single average location error quantity. Table 3.1 shows the average $2 \sigma$ uncertainty ellipse radius for the ten sensors, as computed from the $\mathrm{CRB}$, and also the estimated average uncertainty ellipse computed from Monte-Carlo experiments. The Monte-Carlo results are found by estimating $\alpha_{r}$ for each of the 200 Monte-Carlo experiments. We also compute the true $\alpha_{r}$ vector by transforming the true $\alpha$ vector so that its centroid is zero. In each Monte-Carlo experiment we compute the average distance from each sensor to its corresponding true relative location, then average this distance over all Monte-Carlo experiments. We see that the results agree, and the average uncertainty radius is approximately $0.45 \mathrm{~m}$ for this example. Not shown in the figure are the sensor orientation angles and their estimates; the $2 \sigma$ angle error bound, predicted from the $\mathrm{CRB}$, is $1.8^{\circ}$ for each sensor, and the Monte-Carlo estimates are close to this value as well.

We also estimate the absolute location and the average location error. In this case we compute $\hat{\alpha}$ for each of the 200 Monte-Carlo experiments and then compute the average distance from the true locations as above. We compare this average $2 \sigma$ error distance to the corresponding average error computed from $\operatorname{cov}(\hat{\alpha})$ in equation (3.31). These results are 
also shown in Table 3.1. We see that the absolute $2 \sigma$ error is about 5 meters, approximately $1 / 4$ the prior $2 \sigma$ uncertainty of 20 meters for each sensor. The theoretical variance of $5.19 \mathrm{~m}$ in the table is typical; in another simulation we computed the theoretical $2 \sigma$ absolute distance error for 200 choices of true sensor locations and found the average of these 200 distances to be $5.17 \mathrm{~m}$.

This experiment shows that relative location calibration of the sensors to errors well less than 1 meter is possible. In addition, even with weak prior information of sensor locations, as might be available from aimpoints for sensor placement, absolute location uncertainties on the order of 5 meters can be obtained. It is significant that the relative location errors are small - this means that beamforming or source location and tracking can be expected to have high accuracy. The absolute location corresponds to uncertainty only in the translation or rotation of the entire sensor network. While this leads to a corresponding uncertainty in, for example, a target being tracked by the network, the uncertainty lies largely in the translation and rotation of the track. Other available information may be used to further correct for this location and translation error. If a track is found to be slightly translated and rotated from a known road, for example, the absolute orientation sensor network can be correspondingly adjusted.

\subsubsection{Field Test Results}

We present the results of applying the auto-calibration procedure to an acoustic source calibration data collection conducted during the DUNES test at Spesutie Island, Aberdeen Proving Ground, Maryland in September 1999. In this test, four acoustic sensors are placed at known locations 60-100 $\mathrm{m}$ apart as shown in Figure 3.5. Four acoustic source signals are also used. Exact ground truth locations of the calibration sources are not known, but it is 
known that each source is within approximately $1 \mathrm{~m}$ of a sensor. Each source signal is a series of bursts in the $40-160 \mathrm{~Hz}$ frequency band. Time-aligned samples of the sensor microphone signals are acquired at a sampling rate of $1005.53 \mathrm{~Hz}$. Times of arrival are estimated by cross-correlating the measured microphone signals with the known source waveform, and finding the peak of the correlation function. Only a single microphone signal is available at each sensor, so while TOA measurements are obtained, no DOA measurements are available. Figure 3.5 shows the ML estimates of the relative sensor and source location, as compared to the known relative locations of the sensors. The location errors of sensors $\mathrm{A} 1, \mathrm{~A} 2, \mathrm{~A} 3$, and $\mathrm{A} 4$, are $0.11 \mathrm{~m}, 0.50 \mathrm{~m}, 0.30 \mathrm{~m}$ and $0.50 \mathrm{~m}$, respectively, for an average error of $0.35 \mathrm{~m}$. In addition, the source location estimates are on average about $1.3 \mathrm{~m}$ away from the sensor locations, consistent with our ground truth information. It is noted for this experiment that no prior aimpoint information was given for the positions other than the ground truth. Thus, absolute calibration can only be achieved if knowledge of some of the parameters is assumed. This case is studied in the previous chapter.

\subsection{Conclusions}

We have presented a procedure for calibrating the locations and orientations of a network of sensors. The calibration procedure uses source signals that are placed in the scene and computes sensor and source unknowns from estimated time-of-arrival and directionof-arrival estimates obtained for each source-sensor pair. These measurements can be used to compute a maximum likelihood estimate of the relative sensor and calibration source locations, along with the relative orientation of the sensors. Absolute sensor calibration is obtained by using these measurements along with probabilistic prior location information 


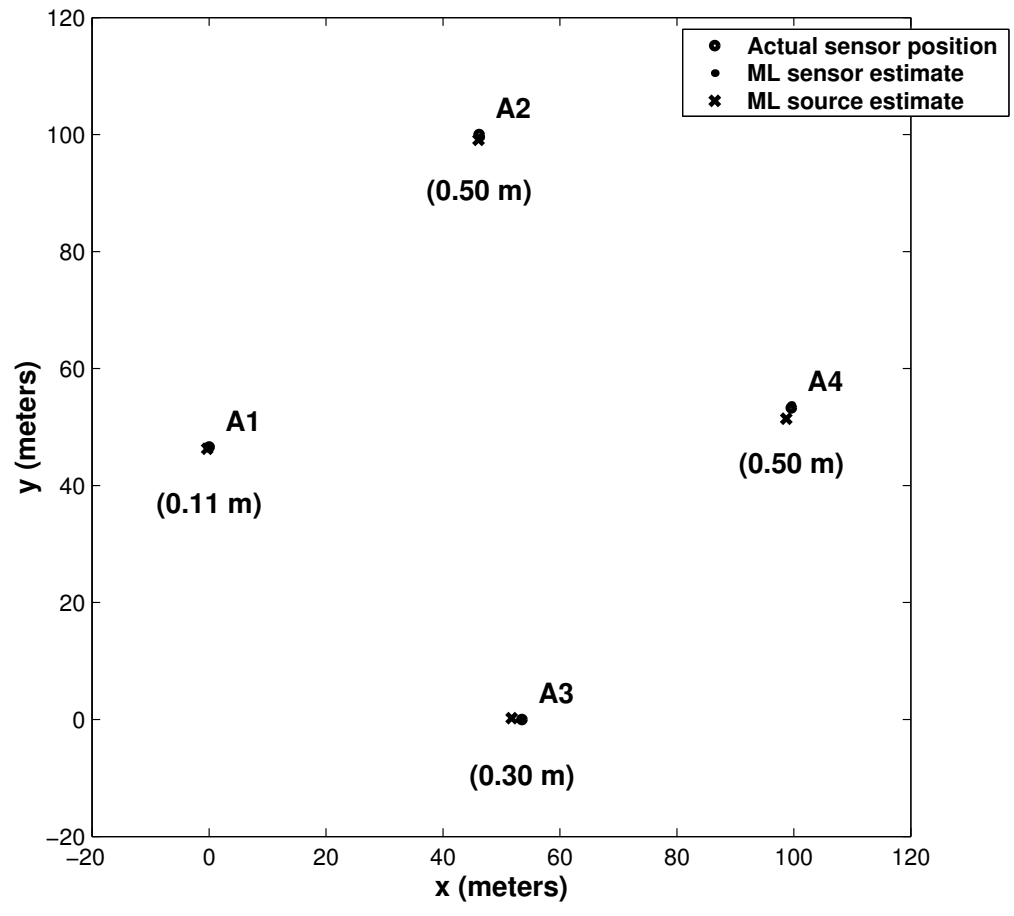

Figure 3.5: Actual and estimated sensor locations, and estimated source locations, using field test data. Error distances between actual and estimated sensor locations are shown in parentheses. 
of some of the sources. A maximum a posteriori calibration solution is proposed in this case.

An analytical expression for the Cramér-Rao lower bound on the relative sensor calibration error covariance matrix is presented. In addition, a high measurement SNR expression for the posterior error covariance of the absolute calibration method is derived. Experimental results on synthetic and measured data validate the theoretical expressions and show that two-standard-deviation relative location accuracies of less than 1 meter can be obtained. Absolute location calibration two-standard-deviation uncertainty of approximately 5 meters was obtained for the cases considered.

The calibration procedure requires low sensor communication and has reasonable computational cost. The algorithms require low communication overhead as each sensor needs to communicate only two real values to the CIP for each source signal it detects. Computation of the calibration solution takes place at the CIP. For the synthetic examples presented the calibration computation takes on the order of 10 seconds using Matlab on a standard personal computer. For the field test data, computation time was less than 1 second. 


\section{CHAPTER 4}

\section{GRAPHICAL USER INTERFACE}

\subsection{Program Description}

A Graphical User Interface (GUI) program was developed to easily demonstrate the calibration methods given in Chapters 2 and 3. The program is the next version of the GUI developed by Dushyanth Krishnamurthy [31]. Substantial edits have been made to the previous code for increased operability and stability, and functions have been added to show new concepts. The GUI was written in Matlab and is intended for use in version 6.0. Later versions of Matlab should work based on the concept of backwards compatibility, but older versions will not work.

The front panel of the GUI is shown in Figure 4.1. The large grid in the display is the main axes where the majority of user interaction occurs. A zoom-in window is shown just to the right of the main axes and is used when the user desires a closer look at sensor and source calibration. Immediately below the zoom-in window is the information box where the user sees position estimates and summary information. The lower left box contains buttons which are used in progression to create a sensor/source map and to calculate accuracy bounds and position estimates. An instruction box is located in the lower central area, and 


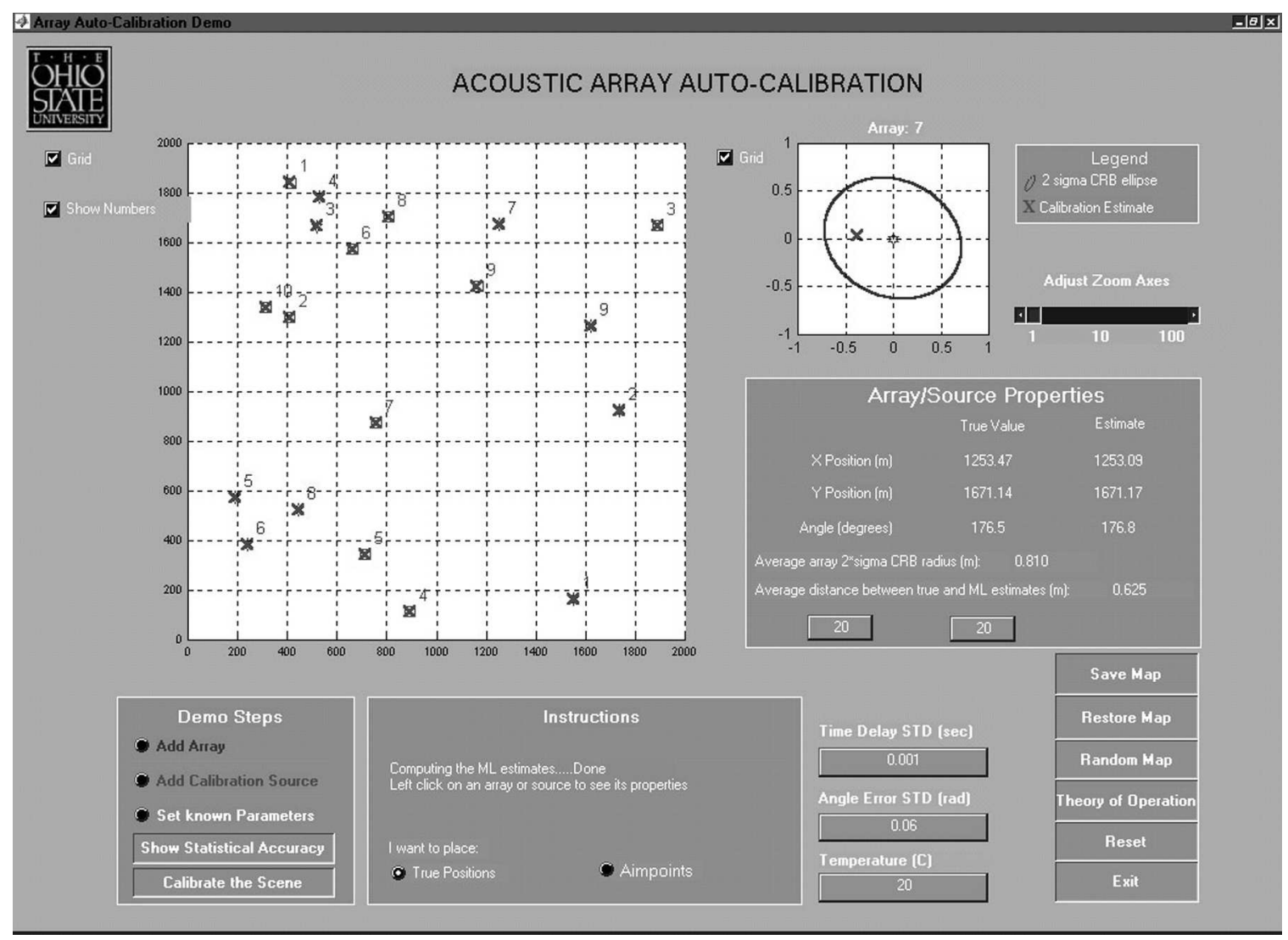

Figure 4.1: A screen shot of the auto-calibration demonstration's main display.

various self-explanatory parameter boxes and program buttons are located to the right of this box.

\subsection{Sensor and Source Placement and Initialization}

The user begins by deciding which method of absolute localization is desired. If some parameters will be considered as being known, the user should select the "True positions" radio button in the information box. We will refer to this as "Mode 1." If the user wants to place aimpoints with known probability density function, the user should select the "Aimpoints" radio button. This is referred to as "Mode 2." 
To place sensors on the main axes, the user should select the "Add array" radio button. In Mode 1 , by clicking on the main axes, the user will create an array at that location. If the user is in Mode 2, the user will generate a circle around that point with the radius of the circle equal to twice the standard deviation of the prior probability density function. The probability density function is Gaussian, and the user sets the desired standard deviation of this density function before clicking on the main axes. Sources are placed in a similar fashion as the placement of arrays was described after selecting the "Add Source" button.

If the user is in Mode 1, sensor and source parameters can be set as being known. The user first selects the "Set Known Parameters" radio button. Sensor location and orientation parameters can be set as known or unknown by right-clicking on each array. Similarly, the user can set the source location and emission time as being known or unknown by right-clicking on a source. The sensor's and source's initial values are initialized as being unknown. If the user is in Mode 2, parameters cannot be set as being known. At all times, the user can delete an array or source by right-clicking on the object and selecting "Delete." Also, sensors and sources can be moved by left-clicking on the object with a mouse, dragging it to the desired location, and then releasing the mouse button.

\subsection{Cramér-Rao Bound and Maximum Likelihood Estimation}

The user can calculate the Cramér-Rao Bound (CRB) on location accuracy. By pressing the "Show Statistical Accuracy" button, the CRB is generated and two standard deviation ellipses are plotted around each sensor and source on the main axes. The user can click on any sensor or source and a copy of that object will be displayed in the zoom-in window. The axes of the zoom-in window can be adjusted by a sliding control, enabling the user to view the CRB at a desired level of detail. 
To calculate the maximum likelihood (ML) estimate, the user presses the "Calibrate" button. First, the actual time-of-arrival and direction-of-arrival values are found using the actual positions of the sensors and sources. Gaussian noise is added to these values to simulate noisy measurements. The amount of noise added is determined by two parameters: Time Delay and Angle Error standard deviations. These parameters can be modified by the user as desired on the GUI. The ML sensor and source location estimates are displayed on the main axes once estimation is complete. By clicking on any sensor or source, the user can view the ML estimate for that object in the zoom-in axes.

Once the CRB and ML estimates are calculated, the user can click on any sensor or source to view the actual and estimated values in the parameter box on the GUI. In addition, the average two standard deviation ellipse radius and average ML estimate error for the sensors can be viewed.

\subsection{Additional Features}

Several user-friendly features have been built into the GUI. The user can save and restore a map. This enables a user to repeat experiments on a single map configuration between Matlab sessions. The user can have the GUI generate a random sensor/source scene. Also, the user can view a demonstration of how the localization algorithm works by selecting the "Theory of Operation" button. 


\section{CHAPTER 5}

\section{CONTRIBUTIONS AND FUTURE WORK}

\subsection{Conclusions}

In this thesis we provide a solution to the self-calibration problem for a network of sensors. We study the existence of solutions by finding the minimum number of sensors and sources required for self-calibration. A method for calculating the maximum likelihood estimate is developed. The accuracy with which we can estimate the sensors' parameters is given by a Cramér-Rao Bound calculation. We find that the maximum likelihood estimation method is statistically efficient for high signal-to-noise ratio measurements.

This thesis also explores relative and absolute calibration. We provide methods for absolute calibration when either we know several parameters, or we have prior knowledge about the positions of the sensors and sources. In the latter case, we provide maximum $a$ posteriori location estimates. We also provide methods to determine the estimation accuracy in this case through use of a Cramér-Rao Bound calculation.

Lastly, we describe a flexible and easy-to-use tool that was developed to demonstrate our algorithms. Sensors and sources can be placed in a scene, and by using simulated noisy 
DOA and TOA measurements, we can estimate sensor and source parameters. The graphical user interface allows the user to visually examine calibration estimates and accuracy bounds on relative and absolute calibration.

\subsection{Ideas for Future Work}

Several extensions to the work would be interesting topics for further investigation. First, our problem constrained the sensors and sources to be placed on a plane. Many real-world applications necessitate sensor placement and calibration in three-dimensional space. An additional set of parameters would have to be estimated in this case.

Secondly, we have assumed that calibration signals radiate perfectly from each source. Various atmospheric conditions can make this assumption invalid. For instance, temperature variations cause the speed of sound to change. If a temperature gradient existed in the region of sensor and source deployment, we would have to model the speed of sound as a function of position. Also, wind and sound reflections increase the difficulty of the problem.

Finally, an investigation into how parameter estimation would change if sensors and sources are co-located would be interesting. We can assume in some cases that each sensor would also contain a sound source. In this case source signal emission times would be known, and the number of parameters needed to be estimated would decrease by half due to sensor and source positions being identical for corresponding pairs of sensors and sources. 


\section{BIBLIOGRAPHY}

[1] D. Estrin, L. Girod, G. Pottie, and M. Srivastava, "Instrumenting the world with wireless sensor networks," in Proceedings of the International Conference on Acoustics, Speech, and Signal Processing, vol. 4, (Salt Lake City, UT), pp. 2033-2036, May 7112001.

[2] G. Pottie and W. Kaiser, "Wireless integrated network sensors," Communications of the ACM, vol. 43, pp. 51-58, May 2000.

[3] S. Kumar, F. Zhao, and D. Shepherd, "Collaborative signal and information processing in microsensor networks," IEEE Signal Processing Magazine, vol. 19, pp. 13-14, March 2002.

[4] N. Srour, "Unattended ground sensors: a prospective for operational needs and requirements," tech. rep., Army Research Laboratory, Adelphi, MD, October 1999.

[5] A. Parker, "Sensing for danger," Science and Technology Review, pp. 11-17, July/August 2001.

[6] http://www.1-3com.com/cs-east/programs/tactical/rembass. html. L-3 Communications, Inc.

[7] http://www.xbow.com/Industry_solutions/Environment.htm. Crossbow Technology, Inc.

[8] B. Friedlander and A. J. Weiss, "Direction finding in the presence of mutual coupling," IEEE Transactions on Antennas and Propogation, vol. 39, pp. 273-284, 1991.

[9] N. Fistas and A. Manikas, "A new general global array calibration method," IEEE Transactions on Acoustics, Speech and Signal Processing, vol. 4, pp. 553-556, 1994.

[10] B. C. Ng and C. M. See, "Sensor-array calibration using a maximum-likelihood approach," IEEE Transactions on Antennas and Propogation, vol. 44, pp. 827-835, June 1996.

[11] B. Flanagan and K. Bell, "Improved array self calibration with large sensor position errors for closely spaced sources," pp. 484 -488, March 2000. 
[12] N. Bulusu, J. Heidemann, and D. Estrin, "GPS-less low-cost outdoor localization for very small devices," IEEE Personal Communication, vol. 7, pp. 28-34, October 2000.

[13] A. Savvides, F. Koushanfar, A. Boulis, V. Karavas, M. Srivastava, and M. Potkonjak, "Location discovery in ad hoc wireless networks," tech. rep., Networked and Embedded Systems Laboratory, UCLA, June 2000.

[14] V. Cevher and J. H. McClellan, "Sensor array calibration via tracking with the extended kalman filter,” (College Park, MD), pp. 51-56, March 20-22 2001.

[15] K. Yao, R. E. Hudson, C. W. Reed, D. Chen, and F. Lorenzelli, "Blind beamforming on a randomly distributed sensor array system," Journal on Selected Areas in Communications, vol. 16, pp. 1555-1567, October 1998.

[16] L. Kaplan, L. Q. Le, and P. Molnár, "Maximum likelihood methods for bearings-only target localization,” vol. 5, (Salt Lake City, UT), pp. 3001-3004, May 7-11 2001.

[17] C. Savarese, J. Rabaey, and J. Beutel, "Locationing in distributed ad-hoc wireless sensor networks," in Proceedings of the International Conference on Acoustics, Speech, and Signal Processing, vol. 4, (Salt Lake City, UT), pp. 2037-2040, May 7-11 2001.

[18] A. Savvides, C. C. Han, and M. B. Srivastava, "Dynamic fine-grained localization in ad-hoc wireless sensor networks," in Proceedings of the International Conference on Mobile Computing and Networking (MobiCom) 2001, (Rome, Italy), July 2001.

[19] N. Bulusu, D. Estrin, L. Girod, and J. Heidemann, "Scalable coordination for wireless sensor networks: Self-configuring localization systems," in Proceedings of the Sixth International Symposium on Communication Theory and Applications (ISCTA '01), July 15-20 2001.

[20] J. Pierre and M. Kaveh, "Experimental performance of calibration and directionfinding algorithms," (Toronto, Canada), pp. 1365-1368, 1991.

[21] Y. Rockah and P. M. Schultheiss, "Array shape calibration using sources in unknown locations- part ii: Near-field sources and estimator implementation," IEEE Transactions on Acoustics, Speech, and Signal Processing, vol. 6, pp. 724-735, June 1987.

[22] R. J. Kozick and B. M. Sadler, "Algorithms for localization and tracking of acoustic sources with widely seperated sensors," (Laurel, MD), pp. 205-224, October 17-19 2000.

[23] C. Knapp and G. C. Carter, "The generalized correlation method for estimation of time delay," IEEE Transactions on Acoustics, Speech, and Signal Processing, vol. 4, pp. 320-326, August 1976. 
[24] H. L. Van Trees, Detection, Estimation, and Modulation Theory: Part I. New York: Wiley, 1968.

[25] J. Chaffee and J. Abel, "On the exact solutions of pseudorange equations," Transactions on Aerospace and Electronic Systems, vol. 30, pp. 1021-1030, October 1994.

[26] G. J. McLachan and T. Krishnan, The EM Algorithm and Extensions. New York: Wiley, First ed.

[27] J. C. Chen, K. Yao, and R. E. Hudson, "Source localization and beamforming," IEEE Signal Processing Magazine, vol. 19, pp. 30-39, March 2002.

[28] R. L. Moses, D. Krishnamurthy, and R. Patterson, "A self-localization method for wireless sensor networks," Eurasip Journal on Applied Signal Processing, Special Issue on Sensor Networks. Submitted November 2001.

[29] P. B. van Wamelen, Z. Li, and S. S. Iyengar, "A fast expected time algorithm for the point pattern matching problem," Pattern Recognition. Submitted November 2000.

[30] P. Stoica and T. L. Marzetta, "Parameter estimation problems with singular information matrices," IEEE Transactions on Signal Processing, vol. 49, pp. 87-90, January 2001.

[31] D. Krishnamurthy, "Self-calibration techniques for acoustic sensor arrays," Master's thesis, The Ohio State University, 2001. 